\title{
A Chimera Method for the Incompressible Navier-Stokes Equations
}

\author{
G. Houzeaux ${ }^{1 *}$, B. Eguzkitza ${ }^{1}$, R. Aubry ${ }^{3}$, H. Owen ${ }^{1}$ and M. Vázquez ${ }^{12}$ \\ ${ }^{1}$ Barcelona Supercomputing Center (BSC-CNS), 08034 Barcelona, Spain \\ ${ }^{2}$ Artificial Intelligence Research Institute CSIC (IIIA-CSIC), Bellaterra, Spain \\ ${ }^{3}$ CFD Center, George Mason University, Fairfax, USA
}

\begin{abstract}
SUMMARY
The Chimera method was developed three decades ago as a meshing simplification tool. Different components are meshed independently and then glued together using a domain decomposition technique to couple the equations solved on each component. This coupling is achieved via transmission conditions (in the finite element context) or by imposing the continuity of fluxes (in the finite volume context). Historically, the method has then been used extensively to treat moving objects, as the independent meshes are free to move with respect to the others. At each time step, the main task consists in recomputing the interpolation of the transmission conditions or fluxes. This paper presents a Chimera method applied to the Navier-Stokes equations. After an introduction on the Chimera method, we describe in two different sections the two independent steps of the method: the hole cutting to create the interfaces of the subdomains and the coupling of the subdomains. Then we present the Navier-Stokes solver considered in this work. Implementation aspects are then detailed in order to apply efficiently the method to this specific parallel Navier-Stokes solver. We conclude with some examples to demonstrate the reliability and the application of the proposed method. Copyright (c) 2013 John Wiley \& Sons, Ltd.

Received ...
\end{abstract}

KEY WORDS: Navier-Stokes equations; Chimera method; Incompressible flow; Fractional step methods; Pressure Schur complement system; Parallelization

\section{INTRODUCTION}

Why the Chimera method. The main idea of the Chimera method $[1,2,3,4]$ is to generate independent meshes for the components present in the computational domain (e.g. fuselage, airfoil,

\footnotetext{
${ }^{*}$ Correspondence to: Barcelona Supercomputing Center (BSC-CNS), Dept. Computer Applications in Science and Engineering, Edificio NEXUS I, Campus Nord UPC, Gran Capitán 2-4, 08034 Barcelona, Spain. E-mail: guillaume.houzeaux@bsc.es

Copyright (c) 2013 John Wiley \& Sons, Ltd.
}

Prepared using fldauth.cls [Version: 2010/05/13 v2.00] 
flap, slap, etc.) and to couple them via a coupling strategy in order to obtain a global solution. The appealing characteristics of the Chimera method has enabled many applications, summarized in the following:

- Simplified mesh generation. [3,5] At the time when mesh generators could not handle complex geometries, the Chimera method was proposed as an alternative. Different meshes around the components of the computational domain are generated in an independent way. This enables a great flexibility in the choice of elements in each mesh and to take into account local refinement as the one needed in boundary layers.

- Local refinement. [6, 7] When more accuracy is required in some specific parts of the computational domain, the Chimera method is a smart alternative in codes that cannot handle adaptivity. Local refinement can be achieved by simply putting a refined patch mesh onto the original mesh.

- Moving components. $[8,9,10,11]$ The Chimera method is well suited for treating problems where components are moving (e.g. opening flat). The independent meshes are moved as rigid bodies and the solution is recoupled when suited (e.g. each time step). This recoupling can be very costly and cumbersome when considering parallel solvers on distributed memory machines. However, they enable to maintain the boundary layers and local refinement around the bodies in a natural way.

- Optimization. [12] Another straightforward application is configuration optimization. A series of objects with independent meshes can be moved around without having to remesh the whole computational domain. Then, optimization techniques can be used to find the optimum configuration of the components.

The main advantage of the Chimera method for the last two applications with respect to a complete automatic remeshing stems from the fact that the component meshes move like rigid bodies from one time step/configuration to another. The coupling only affects the vicinity of the interfaces and, if the interfaces are far enough from the body, it is expected that the impact of the coupling is minimum. Here are some examples of Chimera-type grid assembly codes: Sugar [13], Pegasus 
[14], Overture [15], Code_Saturne [16].

The Chimera jargon. When the Chimera Method is considered, the mesh is divided into a background mesh, which covers all the computational domain, and patch or overset meshes attached to the different components (objects) which are located upon the background mesh. First, a proper preprocess is applied to create the interface of the background mesh with the overset meshes. This can be achieved by taking off elements (in Finite Element jargon) of the background mesh located inside the patch meshes to create apparent interfaces between the background and the patches: this is the hole cutting. If the patch meshes are stationary, the elements of the hole can be removed permanently from the mesh definition. Then a domain decomposition (DD) coupling algorithm is carried out in order to obtain a "continuous solution" across the interfaces. The present work is complementary to the one presented in [17], where a DD coupling algorithm is devised for general PDE's. This paper is an extension to the Chimera method applied to the Navier-Stokes equations.

Different implementations. In the literature, all the Chimera methods have a common feature, the hole cutting. They differ in the way the coupling of the background with the patches is carried out. Figure 1 illustrates three options. In the first method shown in Figure 1 (Left), the hole-cutting is created in such a way that a gap between the two subdomains is obtained. Then, the gap is meshed such that a conforming mesh connects the subdomains (see e.g. [6]). This technique is commonly used as a meshing strategy to glue octree meshes to boundary layer meshes(see e.g. [18]). The two main advantages of this method are: on the one hand, it consists of a preprocess technique that can be taken out of the simulation code; on the other hand, the resulting mesh is conforming. The main drawback is that some nodes may be added if the mesh sizes are different, and the meshing can be tricky. The Shear-Slip Mesh Update Method (SSMUM) [19] is a particular case of this method where the nodes on the interfaces are reconnected to the other interface nodes without adding any node in the gap, which restricts the application of the method to meshes of similar sizes 


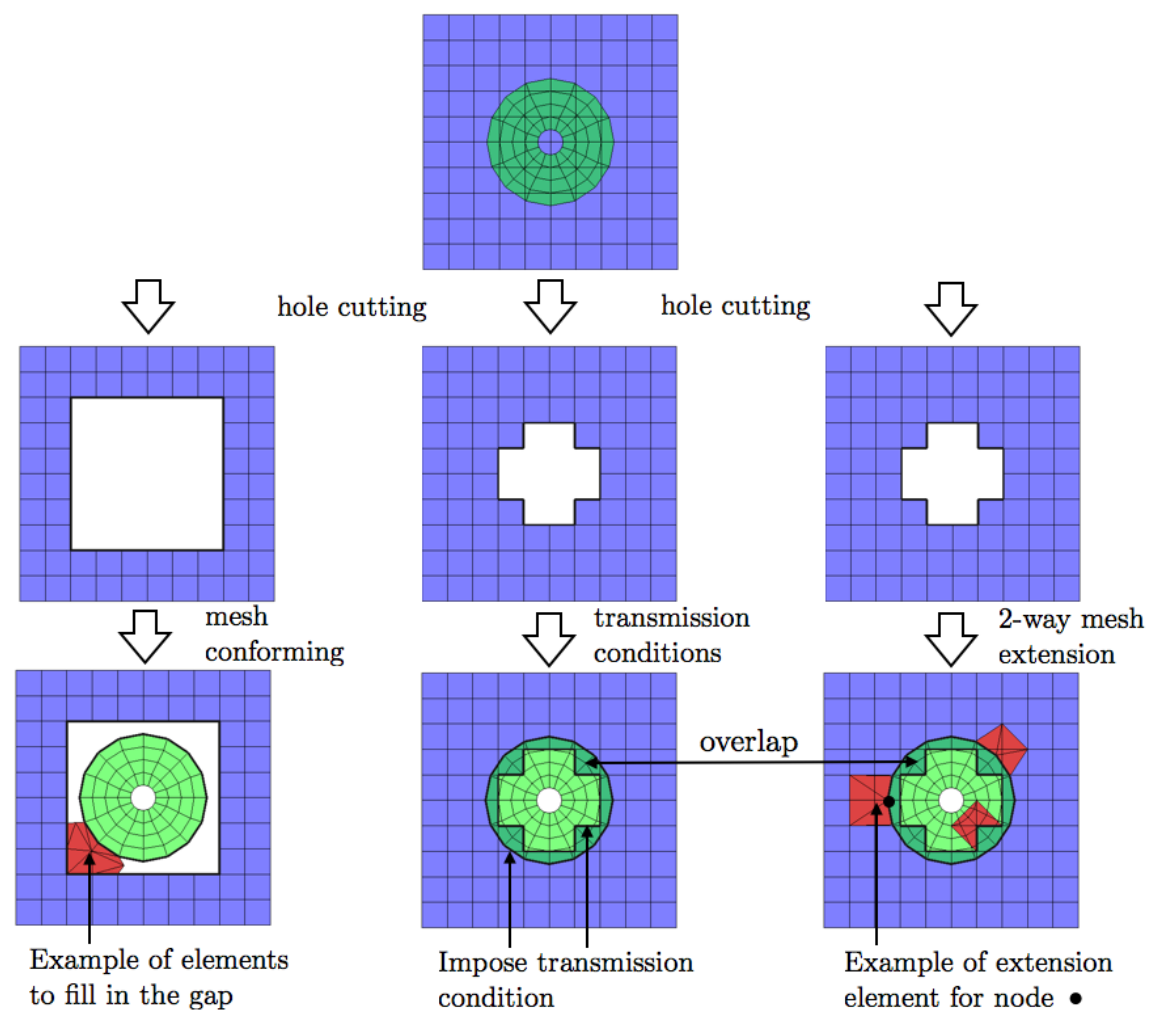

Figure 1. Some Chimera methods.

on both sides of the gap. The Chimera method shown in Figure 1 (Middle) consists in imposing transmission conditions to obtain continuous variables and continuous fluxes across the interfaces. In a finite element context, the transmission condition on one interface could be of Dirichlet type and the other one of Neumann type, to impose continuity of the solution and its flux, respectively. Usually, the coupling is imposed iteratively, imposing an additional iteration loop to the original algorithm [20]. An extensive review of this kind of strategy is the book [21]. In addition, the flux continuity is PDE dependent and a specific implementation would be required for different physics. The last method, illustrated by Figure 1 (Right) is the one proposed in the present work. It consists in connecting one mesh to the other by creating some gluing elements, referred to here as extension elements. The advantages of the method are: it leads to an implicit coupling; it can be applied to any PDE disregarding the physics considered; the method is inherently parallel. It is therefore 
implicit, versatile and parallel.

Present work. The proposed parallel implementation of the Chimera method is valid only for fixed components. In fact, the coupling between the meshes is done as a preprocess, before the mesh partitioning is carried out for parallelization purpose. This is not an inherent restriction of the method but a deliberate choice of the authors, due to the specific requirements of the envisaged applications in this work. It should be mentioned that the parallelization of the Chimera method in a distributed memory context is not an easy task, whenever the method is intended to be implicit. In common explicit implementations of the method, the transmission conditions are usually imposed iteratively so that the subdomains can be solved in a staggered way. In this case, the parallelization is more straightforward as one could use as many parallel instances of the code as subdomains and the only difficulty consists in exchanging of the transmission conditions through MPI. Figure 2 illustrates the differences between an explicit and an implicit coupling in parallel. On the one hand, in the case of the explicit coupling, each subdomain is solved independently with different parallel instances of the code, and the coupling is carried out between these instances in an iterative way; for example, solve 1 , send transmission conditions to 2 , solve 2 , send transmission conditions to 1 , and so on until convergence. In this case, the coupling involves only the right-hand side of the respective algebraic systems. On the other hand, in the implicit case, the transmission conditions are included in the matrix of the algebraic system and the global coupled solution is solved in parallel, regardless the existence of background and patch meshes. In references [22, 23, 24], some implicit strategies are presented in the context of the finite volume method. If the subdomains are moving, the number of degrees of freedom as well as the connectivity in each subdomain vary in time, and the parallel implementation is cumbersome. The proposed method, implemented as a preprocess, can therefore be applied for simplifying the mesh generation, for local refinement and optimization.

The paper is organized as follows. The following section deals with the hole cutting, which leads to the creation of the background interface. The next section describes the coupling strategy. It is 


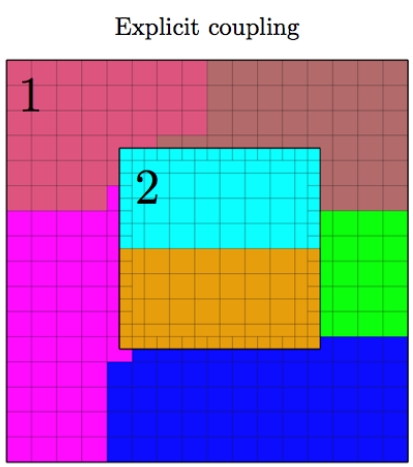

- 1 and 2 are solved in a staggered way, each one in parallel

- Transmission conditions are exchanged iteratively
Implicit coupling

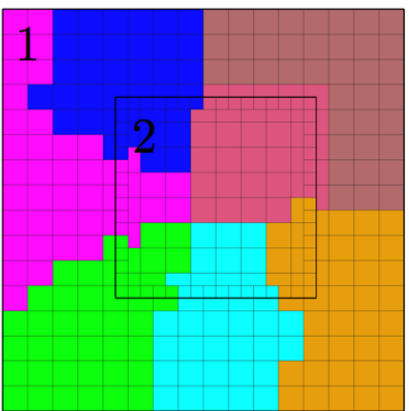

- 1 and 2 are solved as a unique mesh in parallel

- transmission conditions are included in the matrix

Figure 2. Chimera in parallel. (Left) Explicit coupling. (Right) Implicit coupling.

based on an implicit Dirichlet condition imposed in a natural way. Then we present the NavierStokes solver used in this work. It consists of an iterative strategy for the Schur complement system of the pressure (algebraic equivalent of well-known fractional step techniques [25]). Some specific implementation issues related to the Navier-Stokes equations are described in the following section, including parallelization aspects. Finally, some numerical results are presented to assess the reliability of the method.

\section{HOLE CUTTING}

For the sake of clarity we will consider only one patch mesh, although the discussion still holds for multi-components meshes. The hole cutting task consists in removing some elements, the hole elements, from the background mesh. The resulting background mesh is therefore made of the original elements without the hole elements and the interface is the boundary mesh formed by the hole. This interface will be used later on to couple the background with the patch. One key-point when creating the hole is that the boundary of the new mesh is a manifold boundary mesh. 
A triangle mesh is a 2-manifold if it contains neither non-manifold edges nor nonmanifold vertices, nor self-intersections. A non-manifold edge has more than two incident triangles and a non-manifold vertex is generated by pitching two surface sheets together at that vertex such that the vertex is incident to more than one fan of triangles.

More details about the requirements for ensuring mesh validity can be found in [26]. The purpose is to avoid the situation depicted in Figure 3 (Right), where a node (vertex) is connected to three boundary edges and its connected elements (faces in 2D) do not form neither a closed nor an open fan. To ensure that we obtain a manifold mesh, the idea is to mark recursively some candidate

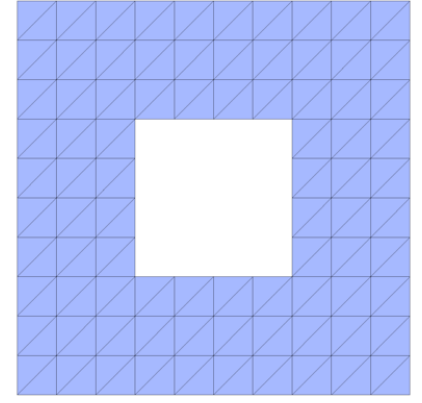

Manifold boundary

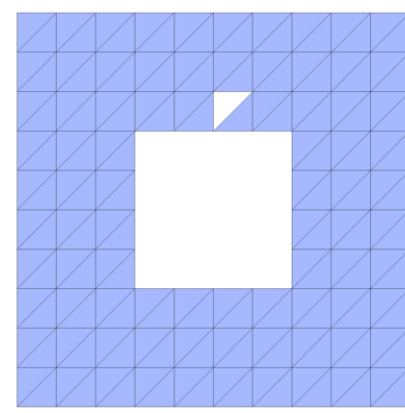

Non-manifold boundary

Figure 3. Manifold and non-manifold mesh after hole cutting.

hole elements, using the common faces as a criterion. Therefore, we will never mark a candidate element if it connects to another marked element trough only an edge or a node. The method consists therefore in two steps:

1. Select the candidate hole elements;

2. Mark the candidate hole elements recursively using the common face criterion.

Candidate hole elements. We propose two methods to select the candidate elements, as illustrated in Figure 4. The first one consists in first marking the elements crossed by the patch interface. The candidate elements are therefore all the elements until the previously marked elements are reached by the recursive algorithm. The second method consists in using the signed distance of the background nodes to the patch interface to identify the hole nodes located inside the patch. In 
this case, the candidate elements are those elements which all nodes are hole nodes. To evaluate the signed distance, we have used a skd-tree strategy, as explained in [27]. Skd-trees are used to find efficiently the signed shortest distance between a point and a surface. In our case, the surface is formed by the patch outer boundary elements.
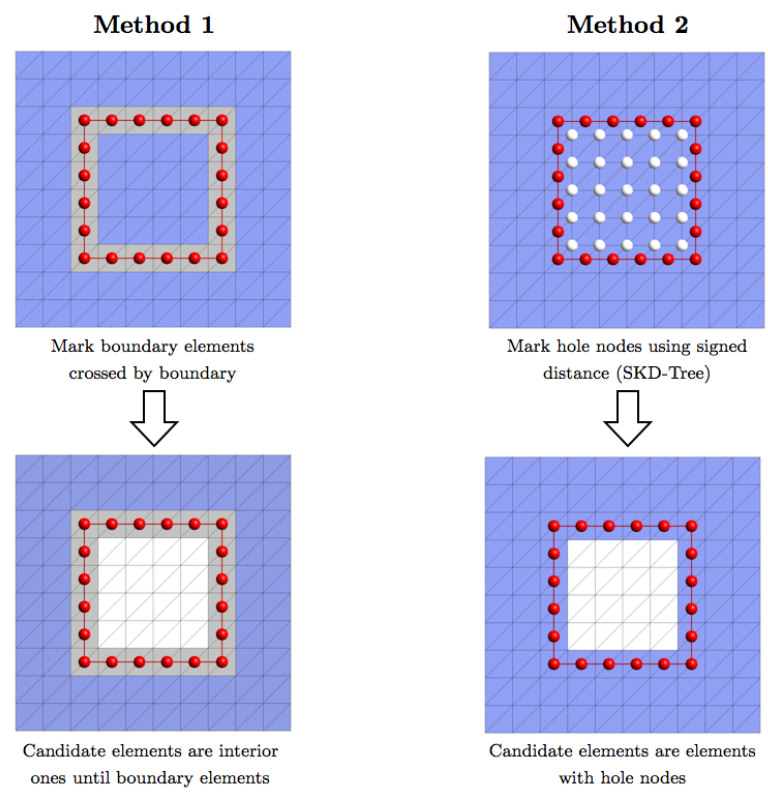

Figure 4. Two methodologies to select hole element candidates. (Left) Marking the elements crossed by patch interface. (Right) Marking the hole nodes using the signed distance to the patch interface.

Hole elements from candidates. Once candidate elements have been selected, a recursive algorithm is executed to create a manifold hole boundary. The seed element of the recursive algorithm is selected near the center of gravity of the hole. Figure 5 represents the recursive algorithm where elements are chosen from top to bottom and black to white. The red element is the seed.

The recursive algorithm is summarized in Algorithm 1. This algorithm can hardly be paralelized and if it is not implemented correctly, this might hamper the overall performance of the Chimera method. We do not give here too much details as the complete algorithm depends greatly upon the data structure at hands. Just to mention that in the current implementation, when marking an element, the associated faces and nodes can also be marked, in order to identify critical edges and 


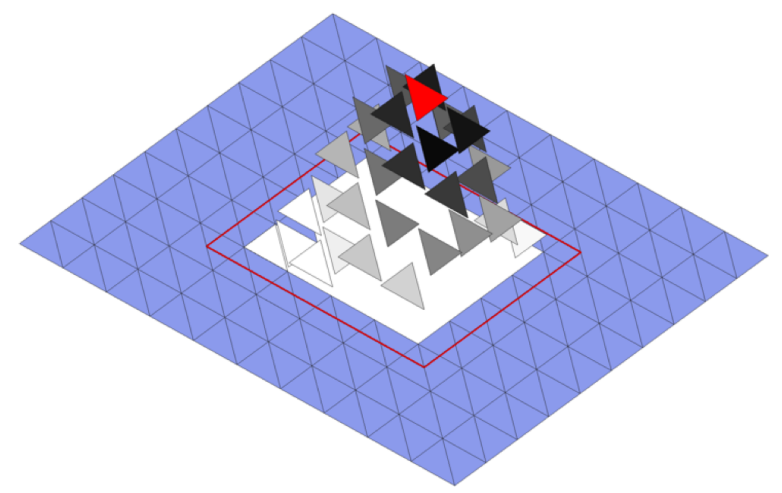

Figure 5. Recursive algorithm to create the hole from the candidate elements. The red element is the seed of the algorithm.

vertices more easily. The algorithm works as follows. From a marked element ielem in the stack, we loop over its neighbors jelem which share common faces. In order to accept and mark the new element jelem, one should check that it only connects to other marked elements kelem through faces. This is necessary to avoid having critical edges or vertices, as illustrated in Figure 6, and thus to obtain a manifold mesh.
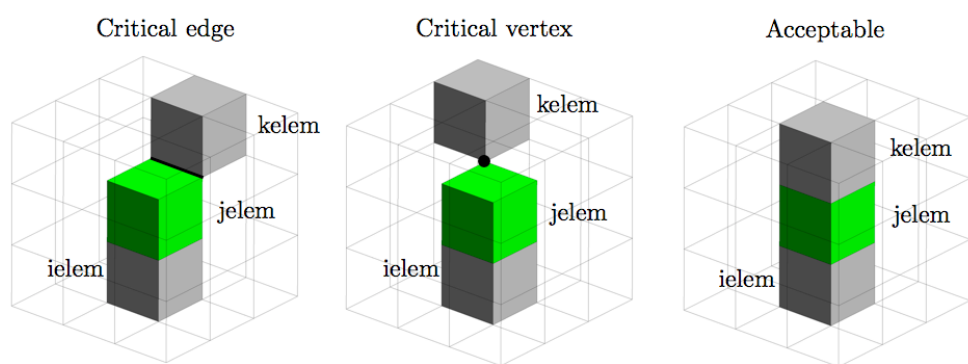

Figure 6. Marking or not a neighbor jelem (green) of ielem (grey) according to the previously marked elements kelem (grey). 


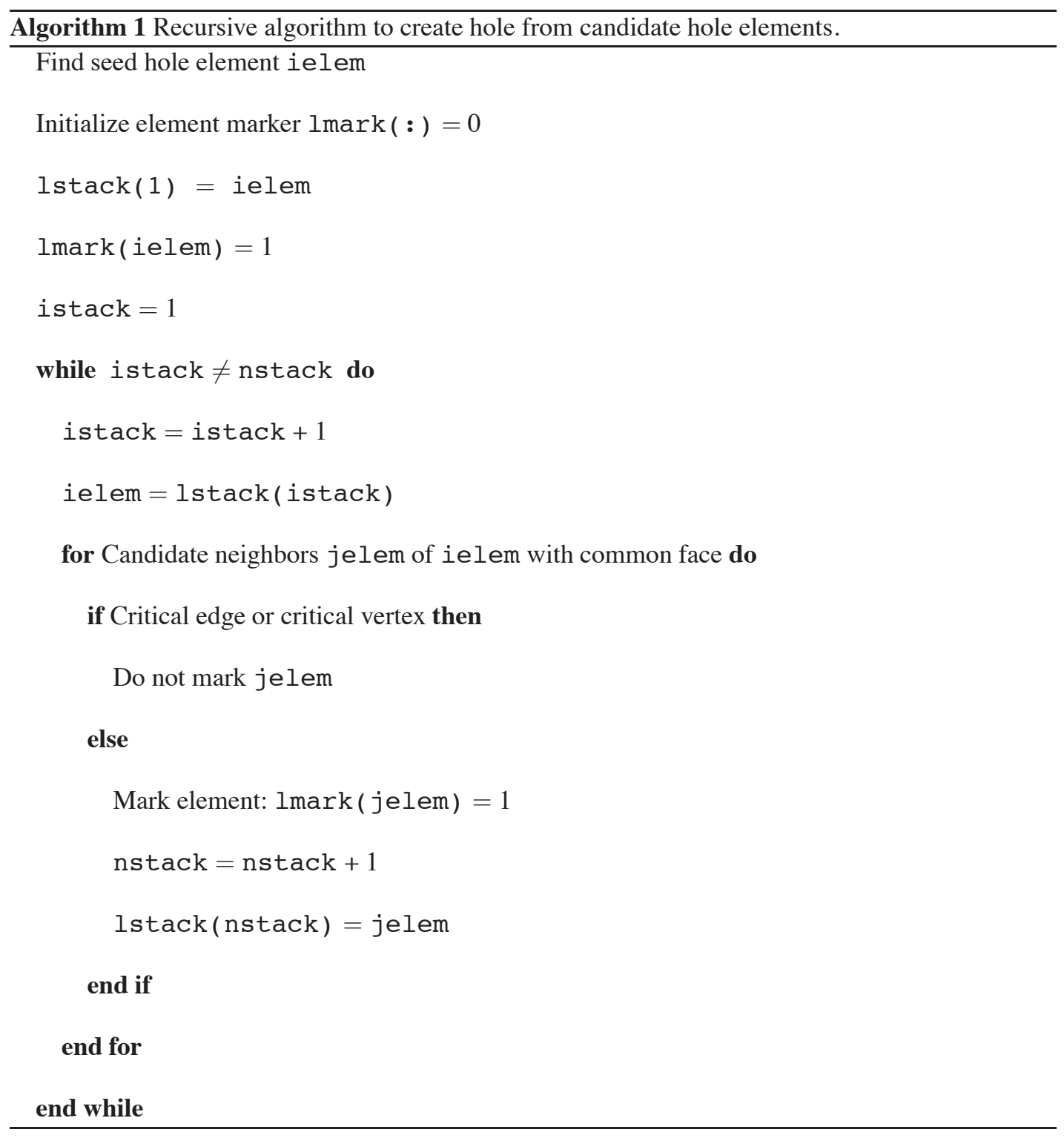

\section{HERMESH COUPLING}

\subsection{Extension elements}

After the hole cutting, the next step of the Chimera method consists in applying a coupling algorithm to couple the PDE solutions that can be obtained on the independent subdomains. The patch will be connected to the background through the nodes of its outer boundary, while the background will be connected to the patch through the fringe nodes of the hole, created previously. The coupling employed here is extensively described in [17] and is referred to as Extension+Dirichlet 
/ Extension+Dirichlet, $(\mathrm{E}+\mathrm{D})^{2}$, while the complete method is referred to as HERMESH (the name comes from the fact that the method Reconnects MESHes). We now recall the main aspects of the coupling by studying a one-dimensional problem. At the end of the section we will illustrate the complete process involving hole cutting and HERMESH coupling using a simple one-dimensional mesh.

Figure 7 illustrates the process at the discrete level, based on the solution of a one-dimensional Poisson equation. On the one hand, the left part of the figure shows a Dirichlet/Dirichlet (D/D) coupling on two overlapping subdomains. On the other hand, the right part of the figure shows the HERMESH method applied in this case to two disjoint subdomains (although the method works also for overlapping subdomains). The top part of the figure shows the meshes and node numberings
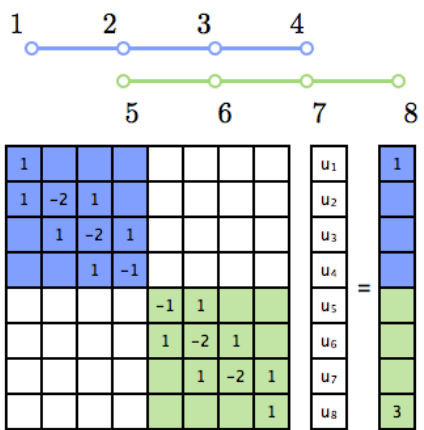

$\Downarrow \mathrm{D} / \mathrm{D}$
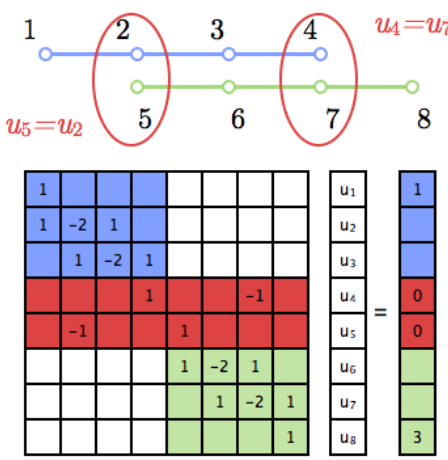

1
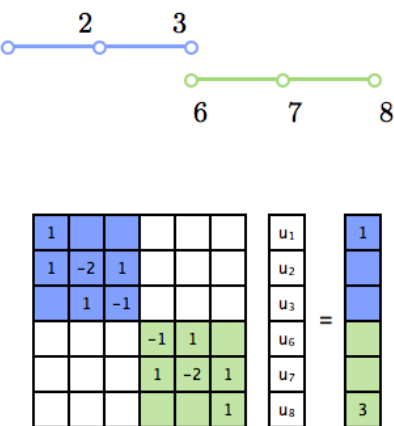

$\Downarrow$ HERMESH
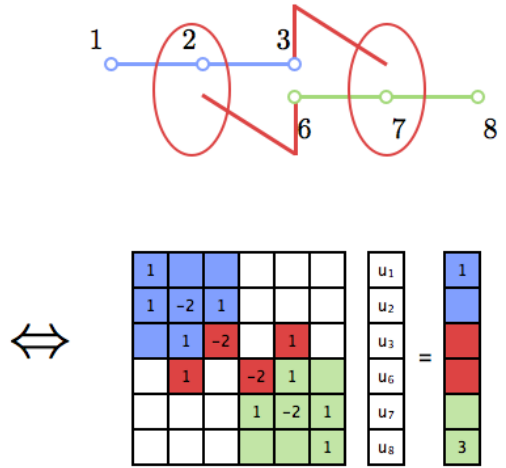

Figure 7. Principles of the HERMESH method and comparison with a Dirichlet/Dirichlet method.

before the couplings. The top matrices are the matrices assembled on these meshes, thus before the couplings. Then, the middle meshes illustrates the couplings. Finally, the bottom matrices are the 
resulting matrices obtained after the D/D and HERMESH couplings. As far as the D/D method is concerned, the coupling is achieved by substituting the rows of nodes 4 and 5 in order to impose $u_{4}=u_{7}$ and $u_{5}=u_{2}$. As far as the HERMESH method is concerned (right part of the figure), the steps of the method are:

- Step 1: Identify interfaces. Obtain a list of the fringe nodes of each subdomains. In this case, the fringe nodes are nodes 3 and 6 .

- Step 2: Extend shape functions. Extend the shape functions of the fringe nodes towards the neighboring subdomains.

- Step 3: Impose Dirichlet condition. Impose the Dirichlet conditions implicitly by choosing the neighbor's nodes to close the support of the shape functions. The shape function of node 3 extends to node 7 , while the shape function of node 6 extends to node 2 .

In practice, Step 2 and Step 3 are carried out at the same time. That is, the extensions of the shape functions of the fringe nodes ( 3 and 6 ) are carried out by adding extension elements to the mesh, painted in red in the figure. In one dimension, the extension element of a fringe node is the element to be created from the boundary (here node 3 and 6) connected to the fringe node to a neighboring subdomain node. If the extension element coincide with an element of the adjacent subdomain (this is the case in this example, elements $2-3$ and 6-7), then this element can be thought as a ghost element, typically used in the paralelization of the finite volume method.

The process in two dimensions is illustrated in Figure 8. In the figure, one of the fringe nodes, represented by a big circle, is connected to two extension nodes of the other subdomain via three extension elements (left part) or two elements (right part). By doing this, we have constructed a global test function with compact support for the fringe node, which overlaps the adjacent subdomain. The process is described in details in the companion paper [17], and we will briefly mention one important aspect concerning the selection of the extension elements. The extension nodes are chosen from a list of properly chosen candidate nodes, such that the associated extension elements fulfill a certain quality criterion. In the case of Figure 8 , the quality criterion would enable one to choose between option 1 and option 2 . 


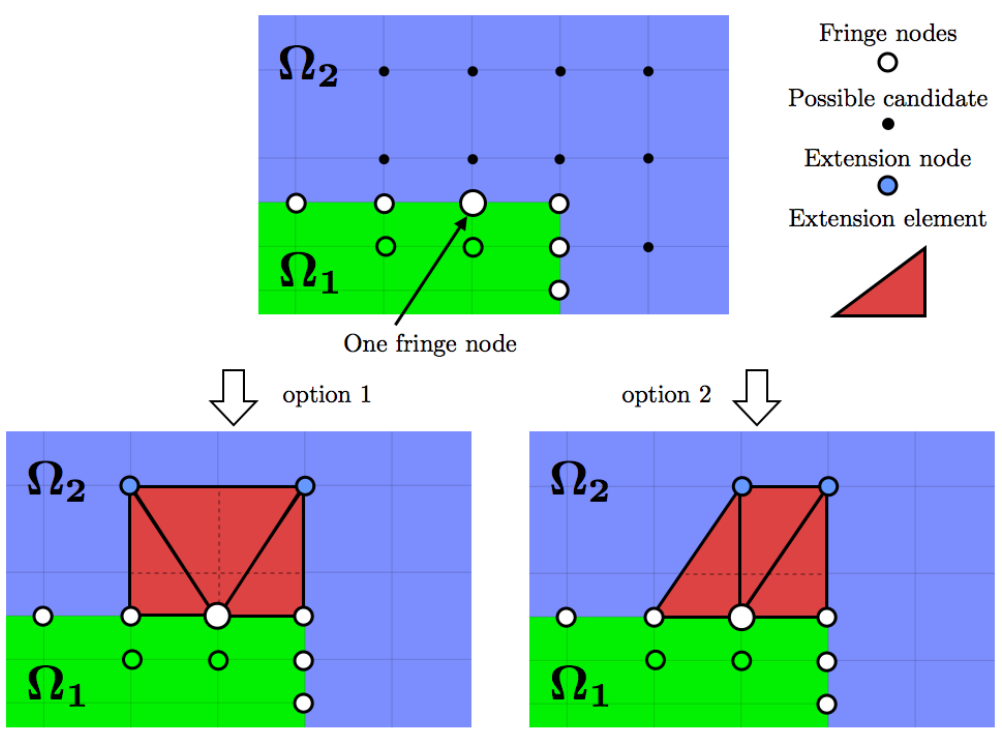

Figure 8. Example of 2D extension elements.

It should be pointed out that the construction of extension elements in three dimensions is not straightforward at all, as described in [17]. The important aspects to take into account when creating the extension elements are:

- Carefully choose the candidate nodes of the neighbor; the list should be long enough to have a flexibility in the choice of candidate extension elements but short enough to control the CPU time;

- Carefully choose a quality criterion to select the possible extension elements coming from the different candidate nodes; In [17], the authors compares the results for different quality criteria;

- If one assumes that the original meshes were generated properly, try to respect the element anisotropy orientation: this is specially true when connecting boundary layer meshes.

The extension elements extend from the boundary connected to the fringe nodes. In $2 \mathrm{D}$ these boundaries are bar elements, while in $3 \mathrm{D}$ the boundaries can be quadrilaterals or triangles. In $2 \mathrm{D}$, we use triangles to extend from the bar elements. In 3D, two extension elements are necessary: pyramids for quadrilaterals and tetrahedra for triangles. Figure 9 illustrates some extension elements in 3D. 
The left part of the figure shows some pyramids to extend from quadrilateral boundary elements; the right part shows some tetrahedra extensions to extend from triangle boundary elements.

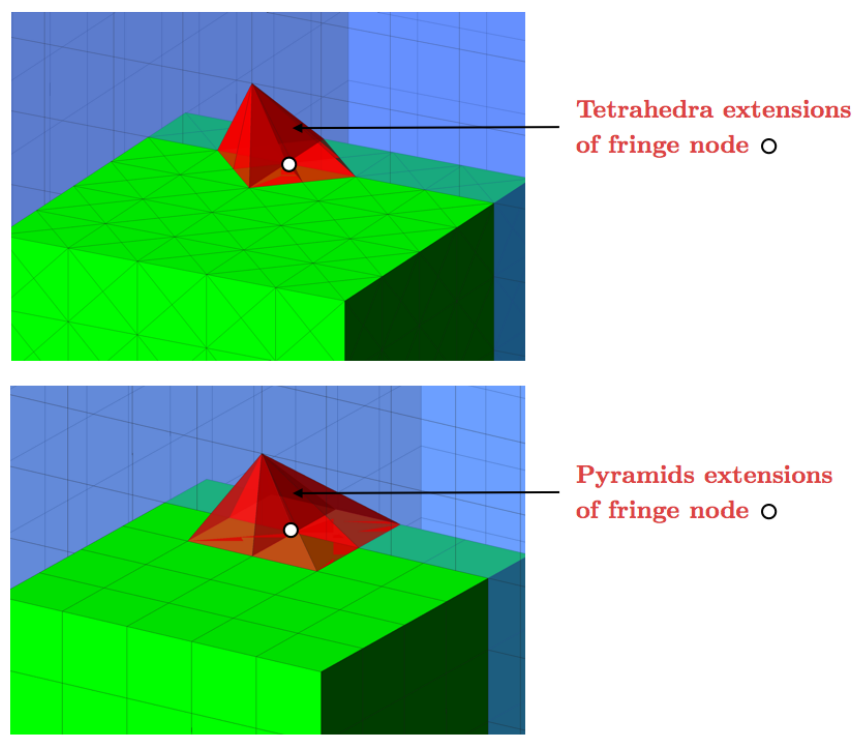

Figure 9. Extension elements in 3D. (Top) Tetrahedra to extend from a triangle boundaries. (Bot.) Pyramids to extend from a quadrilateral boundaries.

\subsection{The crown can be non-conforming}

In order to have a greater flexibility when selecting the best extension elements, we have let the possibility for the crown made by extension elements to be non-conforming. The crown is defined here as the union of extension elements of one subdomain to another. Figure 10 depicts the concept of a crown. It shows the extension elements of three fringe nodes, leading to a non-conforming crown (left part) and to a conforming crown (right part). On the left part, the elements extending from the boundaries connected to the middle fringe node (yellow one) are not coinciding. The resulting test function used to assemble the equation of this node is uniquely defined and its support corresponds to the yellow triangle. However, when assembling the equation of its neighboring fringe nodes, different approximations are used (for example, right red triangle does not coincide with left 

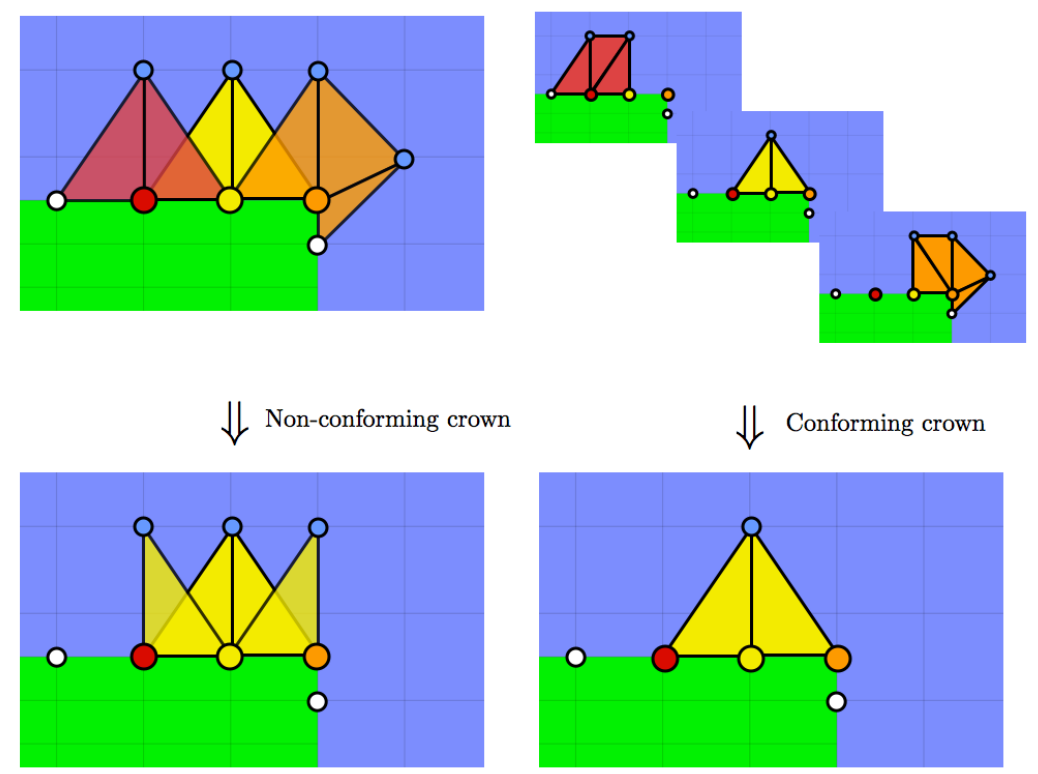

Figure 10. Extension elements in 2D. (Left) Non-conforming crown. (Right) Conforming crown.

yellow triangle). On the other hand, in the conforming case, the extension elements connected to the fringe nodes always coincide. The crown is thus conforming.

\subsection{Hole cutting plus HERMESH coupling}

We now illustrate the complete strategy for setting up the Chimera method using two onedimensional and overlapping meshes, as shown in Figure 11.

The complete strategy consists of the following steps:

- Step 1: Hole cutting. From the top meshes, perform the hole cutting by identifying the hole elements. In the figure, they are elements 4,5 and 6 . We observe that we end up with an overlap between the subdomains. The overlap is the zone comprised between nodes 4 and 10 on the left-hand side, and between nodes 7 and 14 on the right-hand side.

- Step 2: Identify fringe nodes. The fringes nodes are the nodes located on the boundary of the hole of the background (blue subdomain), which are nodes 4 and 7, and the outer boundary of the patch (green subdomain), which are nodes 10 and 14. 

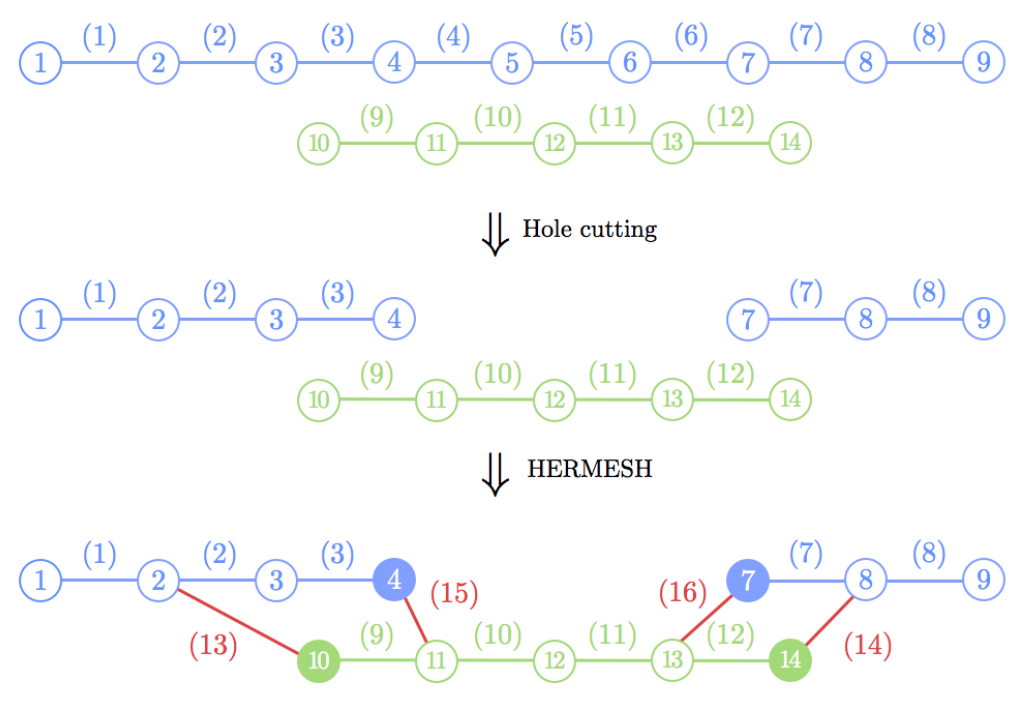

Figure 11. Chimera method: hole cutting plus HERMESH coupling.

- Step 3: HERMESH coupling. Create the extension elements (in red), which are elements 13, 14,15 and 16 , which connect one subdomain to the other. The final overlap is now the zone comprised between nodes 2 and 11 on one side and nodes 7 and 13 on the other side.

\subsection{Equation assembly}

Once the extensions have been created, a very simple implementation is possible. We have to deal with the new types of elements, namely the hole elements and the extension elements.

Hole elements. The governing equations should not be assembled in the hole elements as they are no longer part of the solution process. We can choose to eliminate the hole elements and hole nodes from the mesh definition and apply node and element renumberings before going any further. However, if the Chimera method is implemented to treat moving components, this option is no longer valid. In the present work, we do not eliminate the hole elements and hole nodes to show how the implementation should proceed. Section 5 deals with these implementation aspects. 
Extension elements. The extension elements are attached to a given fringe node and should only be used to assemble the fringe node equation. Therefore, something must be done during the assembly process to avoid the complete contribution of the extension elements. The following strategy treats both the extension and hole elements and is specific to the finite element method.

Let us create an array letyp (nelem) where nelem is the total number of elements (including extensions and holes). Say this array is:

$$
\begin{cases}\text { Normal element: } & \operatorname{letyp}(\text { ielem })=0, \\ \text { Extension element: } & \operatorname{letyp}(\text { ielem })=1, \\ \text { Hole element: } & \operatorname{letyp}(\text { ielem })=-1 .\end{cases}
$$

Let us put the extension node ipoin at the first place in the element connectivity lnods $(1:$ nnode, $1:$ nelem) when we deal with an extension element. For example, if ielem is an extension element of the fringe node ipoin the connectivity would be lnods $(1$, ielem $)=$ ipoin. A classical assembly process in a finite element code (not edge-based) consists in looping over the elements, compute the element matrix and RHS and then scatter the results into the global matrix and RHS. Now, if we deal with an extension, what we need is only to put to zero all the lines of the element matrix and RHS except the first one. In Section 5 we will see how we can use the array letyp to assemble the Navier-Stokes equations.

\section{NAVIER-STOKES SOLVER}

\subsection{Flow equations and Numerical Method}

The flow equations are the incompressible Navier-Stokes equations (viz. [28]). Let $\mu$ be the viscosity of the fluid, and $\rho$ its constant density. The problem is stated as follows: find the velocity $\boldsymbol{u}$ and mechanical pressure $p$ in a domain $\Omega$ such that they satisfy in a time interval

$$
\begin{gathered}
\rho \frac{\partial \boldsymbol{u}}{\partial t}+\rho(\boldsymbol{u} \cdot \nabla) \boldsymbol{u}-\nabla \cdot[2 \mu \boldsymbol{\varepsilon}(\boldsymbol{u})]+\nabla p=\rho \boldsymbol{f}, \\
\nabla \cdot \boldsymbol{u}=0,
\end{gathered}
$$


together with initial and boundary conditions. The term $f$ is the force term, including for example gravity contributions. The velocity strain rate is $\varepsilon(\boldsymbol{u})=\frac{1}{2}\left(\nabla \boldsymbol{u}+\nabla \boldsymbol{u}^{t}\right)$.

The time discretization is based on the trapezoidal rule and the linearization is carried out using the Picard method. The space discretization is based on the variational multiscale method (VMS) and is extensively described in [29], a paper also furnished with a comprehensive reference list on this subject. In any case, the following discussion is independent on the discretization strategy employed. At each time step, the linearized system

$$
\left[\begin{array}{ll}
\mathbf{A}_{u u} & \mathbf{A}_{u p} \\
\mathbf{A}_{p u} & \mathbf{A}_{p p}
\end{array}\right]\left[\begin{array}{l}
\mathbf{u} \\
\mathbf{p}
\end{array}\right]=\left[\begin{array}{l}
\mathbf{b}_{u} \\
\mathbf{b}_{p}
\end{array}\right]
$$

is solved, where $\mathbf{u}$ and $\mathbf{p}$ are velocity and pressure nodal unknowns. Four sub-matrices then arise. Matrix $\mathbf{A}_{u u}$ includes the Galerkin as well as the stabilization terms, like the SUPG-like term and the continuity enforcing term. Matrix $\mathbf{A}_{u p}$ includes the stabilization terms and the Galerkin pressure gradient term. Matrix $\mathbf{A}_{p u}$ includes the velocity divergence operator as well as the part of the pressure stabilization involving the velocity in the momentum residual. Finally, matrix $\mathbf{A}_{p p}$ includes only the pressure stabilization. Note that this sub-matrix is null if div-stab elements are used. When this system is solved in one shot using either a direct solver or an iterative solver with preconditioning, the resulting scheme is referred as a monolithic scheme. The next section explains briefly the split strategy to transform the solution process into a fractional scheme.

\subsection{Algebraic split strategy}

We will obtain in this section an algorithm to solve Equation 3. The steps are summed up in Figure 12. In this subsection, we limit ourselves in describing briefly the algebraic split strategy used to solve the linearized system. The complete development of the algorithm can be found in [30]. We will nevertheless give some details as some of the implementation aspects of the Chimera method depend on this specific algorithm. 


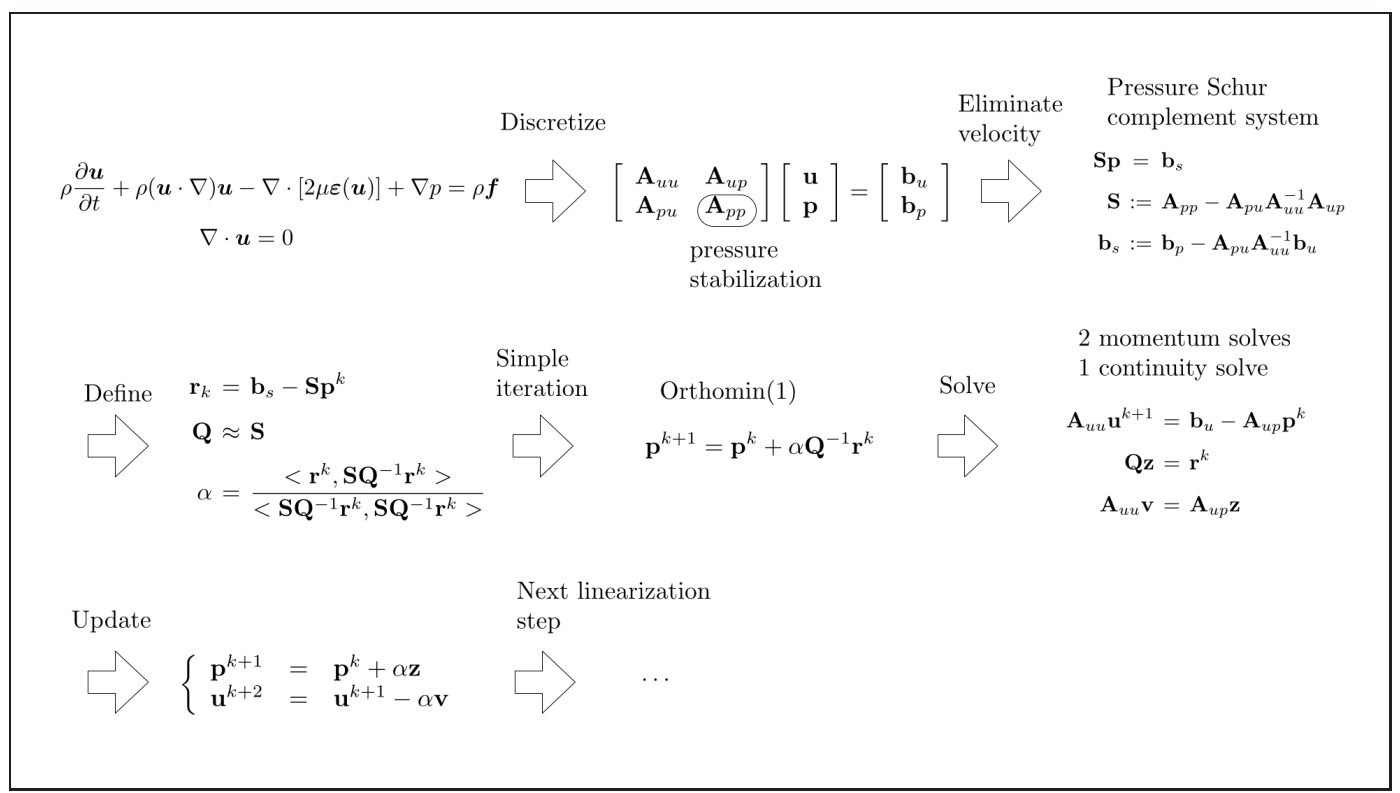

Figure 12. Algebraic split strategy. Orthomin(1) method applied to the pressure Schur complement system.

Pressure Schur complement system. Let us manipulate the matrix system 3 to compute the Schur complement system [31] for the pressure. The Schur complement system is the pressure equation one obtains after eliminating the velocity from the momentum equations:

$$
\begin{aligned}
\mathbf{S p} & =\mathbf{b}_{s}, \quad \text { with }, \\
\mathbf{S} & =\mathbf{A}_{p p}-\mathbf{A}_{p u} \mathbf{A}_{u u}^{-1} \mathbf{A}_{u p}, \\
\mathbf{b}_{s} & =\mathbf{b}_{p}-\mathbf{A}_{p u} \mathbf{A}_{u u}^{-1} \mathbf{b}_{u} .
\end{aligned}
$$

Preconditioned Orthomin(1) iteration. The idea is now to apply a relaxed preconditioned Richardson iteration (also referred to as a simple iteration) method [31, 32] to solve Equation 4. Let $\boldsymbol{r}^{k}$ be the residual of the Schur complement system at iteration $k: \mathbf{r}_{k}=\mathbf{b}_{s}-\mathbf{S p}^{k}$.

We introduce a relaxation parameter $\alpha$ such that the simple preconditioned iteration reads:

$$
\mathbf{p}^{k+1}=\mathbf{p}^{k}+\alpha \mathbf{Q}^{-1} \mathbf{r}^{k}
$$

The preconditioner $\mathbf{Q}$ should approximate $\mathbf{S}$ defined in Equation 5. When $\alpha$ is constant and $\alpha<1$, it is referred to as under-relaxation parameter. When $\alpha$ is constant and $\alpha>1$, it is referred to as the over-relaxation parameter. The Orthomin scheme consists in choosing $\alpha$ in a dynamic way. To do 
so, let us multiply Equation 7 by $\mathbf{S}$ and add $-\mathbf{b}_{s}$ on both sides of the resulting equation. We obtain

$$
\mathbf{r}^{k+1}=\mathbf{r}^{k}-\alpha \mathbf{S Q}^{-1} \mathbf{r}^{k}
$$

Minimizing $\left\|\mathbf{r}^{k+1}\right\|^{2}$ we obtain the following equation for $\alpha$ :

$$
\alpha=\frac{<\mathbf{r}^{k}, \mathbf{S Q}^{-1} \mathbf{r}^{k}>}{<\mathbf{S Q}^{-1} \mathbf{r}^{k}, \mathbf{S Q}^{-1} \mathbf{r}^{k}>} .
$$

The resulting scheme is shown in Algorithm 2.

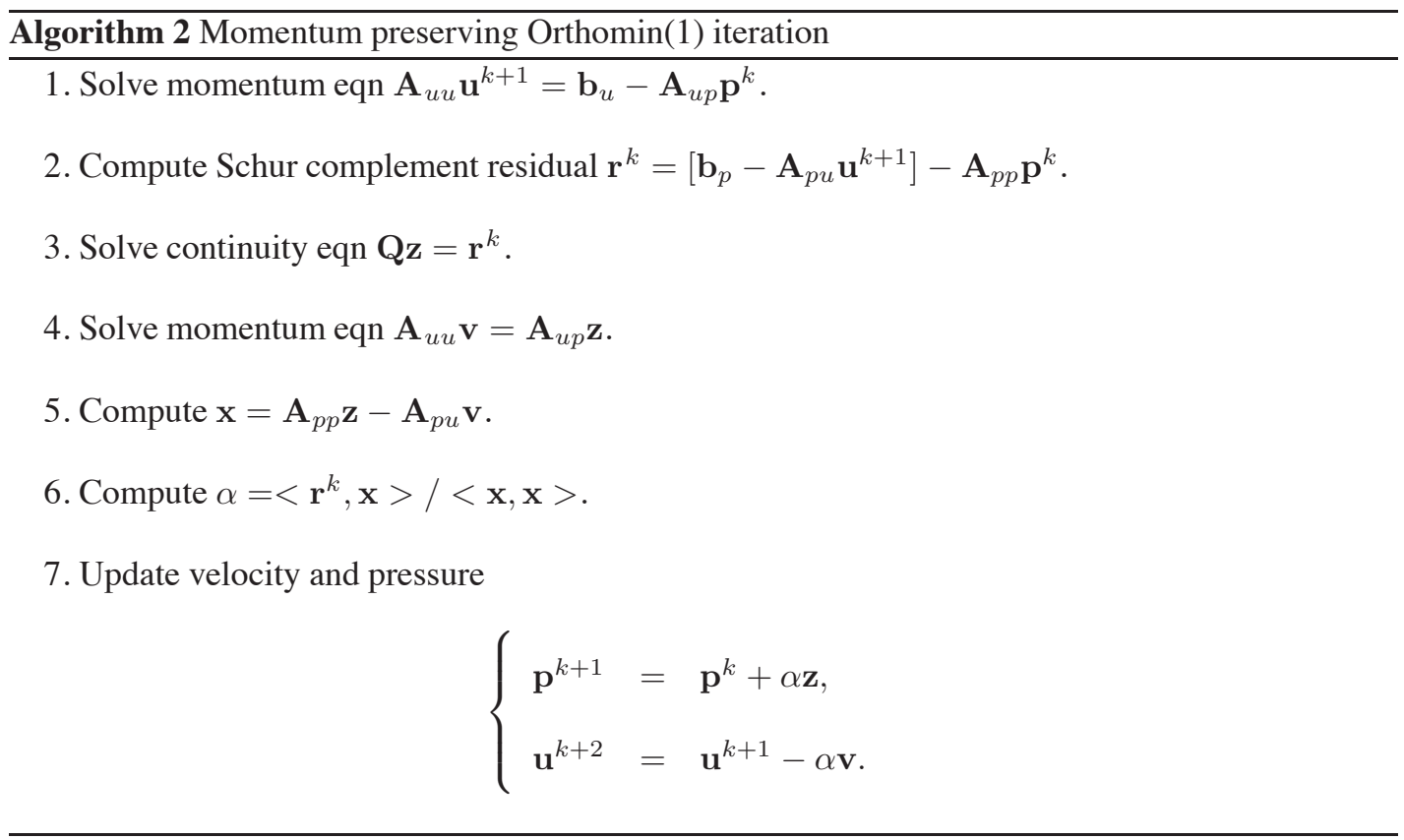

We can check that this algorithm is momentum preserving. It involves two momentum solves, that is one more than classical fractional step techniques. In addition, we can derive from it a continuity preserving version. Figure 13 compares the convergence of the Orthomin(1) with that of the continuity preserving Richardson method (which has tight similarities with classical fractional step methods). The example is a transient LES simulation for which we show the first five time steps. An evident gain in convergence is obtained by the Orthomin(1) method, even though it requires one additional momentum solve. 

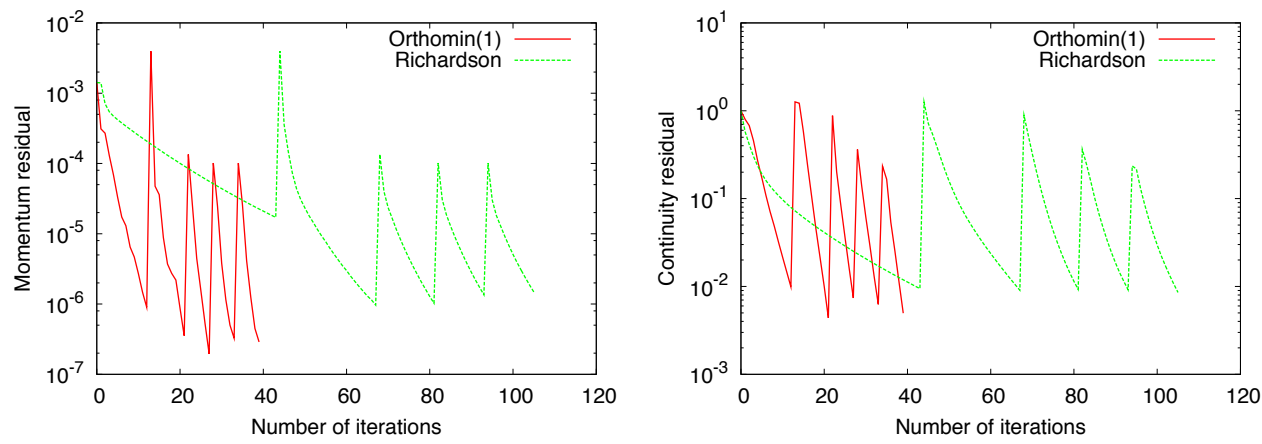

Figure 13. Comparison of convergences of Orthomin(1) and Richardson. (Left) Momentum. (Right) Continuity.

The Schur complement preconditioner $Q$. In the literature, the preconditioner is usually based on the following splitting

$$
\mathbf{Q}=\mathbf{A}_{p p}+\mathbf{P}
$$

where $\mathbf{P}$ approximates the second term of the Schur complement matrix given by Equation 5 such that

$$
\mathbf{P} \approx-\mathbf{A}_{p u} \mathbf{A}_{u u}^{-1} \mathbf{A}_{u p}
$$

Let us introduce the momentum operator $\mathcal{M}$ (after first order time discretization) acting on the velocity as $\mathcal{M}=\rho / \delta t+\boldsymbol{u} \cdot \nabla-\nabla \cdot[2 \mu \varepsilon]$. If we identify $\mathbf{A}_{p u}$ with the divergence operator, $\mathbf{A}_{u u}$ with $\mathcal{M}$ and $\mathbf{A}_{u p}$ with the gradient operator (that is without considering the stabilization contribution to the matrices), $\mathbf{P}$ can be computed from the weak form of Uzawa's operator $-\nabla \cdot \mathcal{M}^{-1} \nabla$ as described in [30]. Using the so-called stabilization parameter $\tau$ as an algebraic approximation of the inverse momentum operator, $\mathbf{P}$ is approximated by the following weak form

$$
\mathbf{P} \Leftarrow \int_{\Omega} \tau \nabla p \cdot \nabla q d \Omega .
$$

The important fact to note here is that the resulting preconditioner $\mathbf{Q}$ is symmetric. 


\section{IMPLEMENTATION ASPECTS}

As shown in Section 3.4, the HERMESH method introduces two new types of elements, namely the hole and extension elements. Some specific aspects must be discussed in order to complete the description of the method.

\subsection{Hole nodes treatment}

We already mentioned that hole elements could be removed from the mesh. However, they are kept in the present implementation. Therefore, as these elements do not participate to the assembly of the global matrix (see Section 3.4), we are left with empty rows in the global matrices and RHS's. These rows correspond to the hole node degrees of freedom. We have basically two alternatives. On the one hand, the hole nodes can be eliminated from the matrix graph (in the CSR format). On the other hand, we have the option to let the matrix as it is; in fact, no free node is connected to a hole node, so any nodal value on a hole node is irrelevant. If this last option is selected, one should only remember to put a non-zero value on the diagonal if a diagonal preconditioner is used in the algebraic solver. The drawback is that useless operations are carried out in the matrix-vector operations of the iterative solvers.

\subsection{Schur complement solver}

One very important aspect is that the HERMESH method presented here does not lead to a directed graph of the nodal connectivity (represented by the CSR format used for the assembly of the matrices). A directed graph connects vertices through edges in a symmetric way, contrary to undirected graphs. More precisely, the graph created by the HERMESH coupling is said to be hybrid, in the sense that it is both directed and undirected. In fact, when a fringe node is connected to an extension node via an extension element, only the equation of the fringe node is assembled in the global system. Therefore, in the matrix, the row of a fringe node has non-zero coefficients in the columns of its extension nodes. However, these extension nodes are not necessarily connected themselves to the fringe node. The graph is therefore locally directed as the relation between a fringe 
node and an extension node is not symmetric. This is illustrated in Figure 14, where the row of the fringe node is colored according to its existing connectivities. On the other hand, the extension nodes are not connected to any node if subdomain 1.

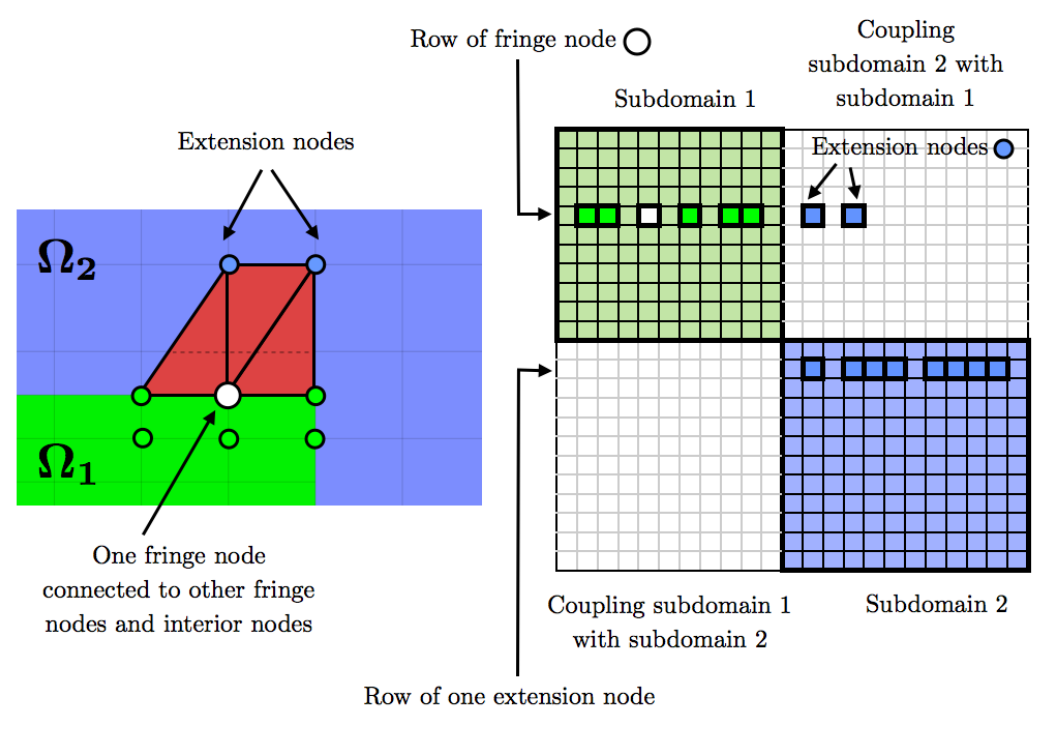

Figure 14. Matrix graph is not fully undirected. A fringe node is connected to extension nodes, but not these ones are not connected to the fringe node.

If the matrix graph has not a symmetric structure, then the resulting matrix will obviously not. Therefore, and unintuitively, the pressure matrix $\mathbf{A}_{p p}$ and the preconditioner $\mathbf{Q}$ are no longer symmetric. $\mathbf{A}_{p p}$ is not an issue. The problems stems from the iterative solution of the Schur complement preconditioner Q presented in Section 4.2 and involved in Step 3 of algorithm 2.

If one wants to use an efficient iterative solver for symmetric systems like the Conjugate Gradient (CG) or the Deflated Conjugate Gradient (DCG), the matrix should be symmetrized. Remember that $\mathbf{Q}$ is only a preconditioner, thus should the solution procedure of the Navier-Stokes equations converge, then it will convergence to the same solution regardless of $\mathbf{Q}$. The symmetrization can be simply achieved. We propose four simple options, illustrated in Figure 15. On the left part of the figure, the element matrix of an extension element is represented for triangle elements ( 3 degrees of freedom per element). In order to obtain a global symmetric system, the extension element matrices 
should be so. The first option, referred to as "all-preconditioner" consists in maintaining the whole element matrix, which is obviously symmetric as it comes from the assembly of Equation 10. The second option, referred to as "symmetrized-preconditioner" consists in assembling only the fringe node row and the fringe node column. In the third option, referred to as "zero-preconditioner", the extension element matrix is not assembled at all. Finally, the fourth option, "diag-preconditioner" consists in retaining only the diagonal. These four options will be compared in Section 6.4. We will show that the best option is the first one, that is the one that retains the whole extension element matrix.

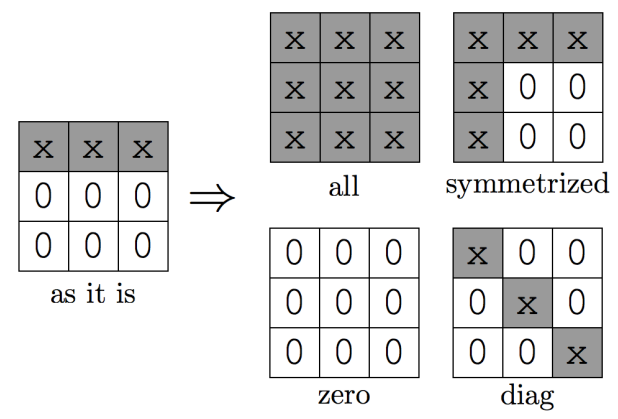

Figure 15. Schur complement solver. Four strategies to obtain a symmetric pressure Schur complement preconditioner.

\subsection{Assembly procedure}

We have just mentioned the way to obtain a symmetric Schur complement preconditioner $\mathbf{Q}$. In the case of the other matrices, i.e. $\mathbf{A}_{u u}, \mathbf{A}_{u p}, \mathbf{A}_{p u}$ and $\mathbf{A}_{p p}$, the same procedure as the one described in Section 3.4 is employed, as $\mathbf{A}_{u u}$ is nonsymmetric in any case (assuming we have convection). The procedure is illustrated in Figure 16, where ndime is the dimension of the problem.

\subsection{Parallelization}

The Chimera method was implemented in Alya system, developed at BSC-CNS. All the details on the code parallelization can be found in [25]. We will only briefly summarize it. The parallelization 


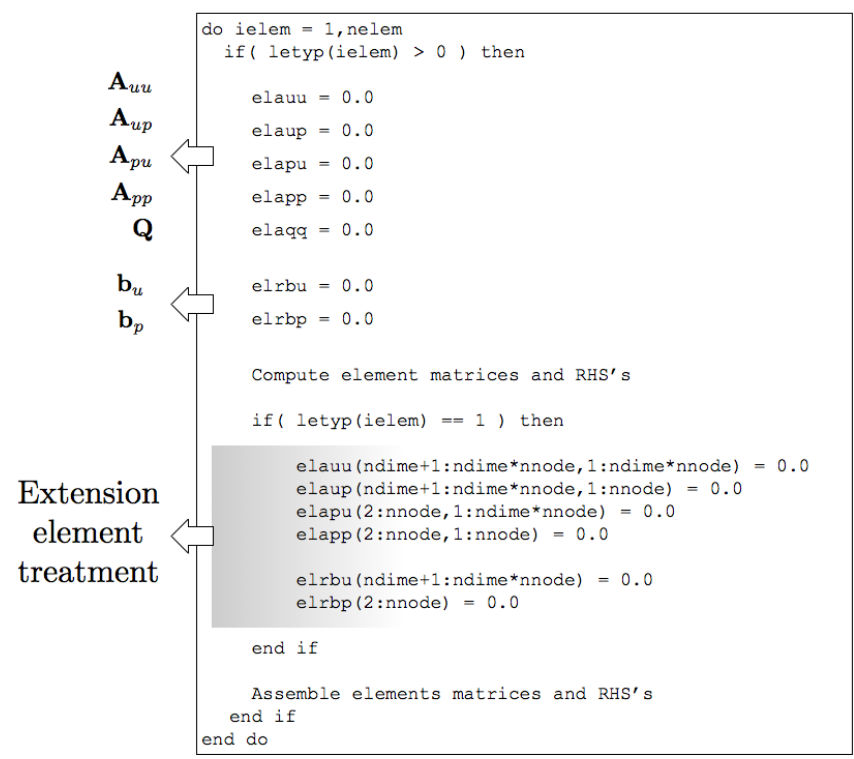

Figure 16. Implementation of the HERMESH method for the Navier-Stokes equations. Extension element is completely assembled for $\mathbf{Q}$.

is based on a master-worker strategy for distributed memory supercomputers, using MPI as the message passing library. The master reads the mesh and performs the partition of the mesh into submeshes, or subdomains, using METIS [33], an automatic graph partitioner. Each process will be in charge of each subdomain, which are the workers. The workers build the local element matrices $\left(\mathbf{A}_{i}\right)$ and right-hand side $\left(\mathbf{b}_{i}\right)$, and are in charge of the resulting system solution in parallel. In the assembling tasks, no communication is needed between the workers and the scalability only depends on the load balancing. In the iterative solvers, the scalability depends on the size of the interfaces between the submeshes and on the communication scheduling.

The momentum and continuity equations are solved with nonsymmetric and symmetric iterative solvers respectively [34]. For the momentum equations, the GMRES or BiCGSTAB are considered while the Deflated Conjugate Gradient (DCG) [35] is the choice for the continuity equation. For most of the applications, the decoupling of the momentum and continuity equations enables the use of the simple diagonal preconditioner to solve both systems, although more complex preconditioners 
like the Linelet [36] may be used for very anisotropic meshes. Along the execution of the iterative solvers, two types of communications are required:

- Global communications via MPI_AllReduce, which are used to compute residual norms and scalar products;

- Point-to-point communications via MPI_SendRecv, which are used when sparse matrixvector products are carried out.

When using more complex solvers like the DCG, additional operations may be required like MPI_AllGatherv functions [37]. In the current implementation of Alya, the solution obtained in parallel is, up to round-off errors, the same as the sequential one all the way through the computation. This is because the mesh partition is only used for distributing work without altering the actual sequential algorithm in any way. This would not be the case if one considered more complex solvers, like the primal/dual Schur complement solvers, or more complex preconditioners, like linelet or block LU.

The next Figure is a schematic flowchart for the execution of a simulation using Alya. The tasks that the master process is responsible for are shown on the left side of the figure with a grey background. The master process performs the first steps of the execution, namely reading the file and partitioning the mesh. Afterwards, the master sends the corresponding subdomain information to each worker process; then the master and the workers enter the time and linearization loops, represented as one single loop. When required, the workers assemble their corresponding left and right-hand sides and then solve the associated linear system exchanging interface information with their neighbours using the MPI function MPI_SendRecv, and using the MPI_AllReduce function for scalar products. In the meanwhile, the master is in charge of the output of the relevant information like system residual.

The difficulty of parallelizing the Chimera method depends on whether the method is intended to be used as a preprocess or for treating moving components. See [9] for a good review about 


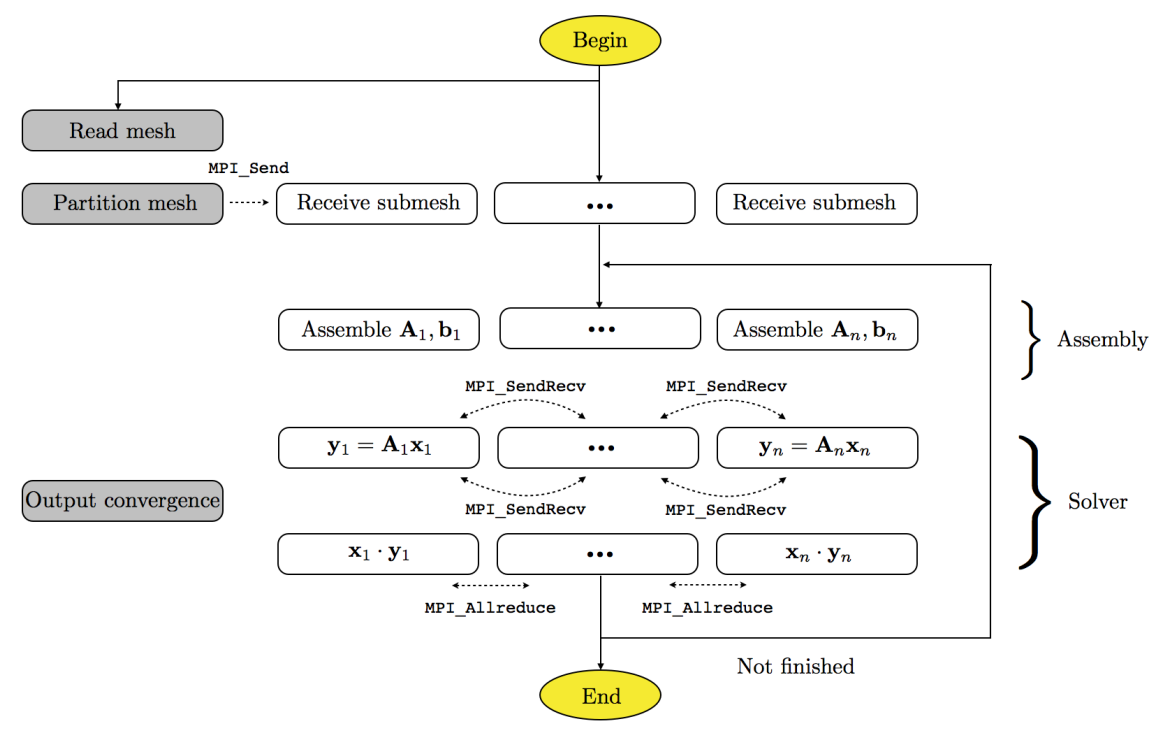

Figure 17. Parallel flowchart. Master (grey) and $n$ workers (white).

this question. Studies on the parallel performance for overset grid can be found in [38, 39, 40] Let us recall the main principles of the proposed method: remove hole elements and hole nodes and create some new elements (extension elements). If one wants to treat moving components, the original mesh should be partitioned before applying the method. Then, for each new configuration, the Chimera principles should be applied; the new elements distributed in some ways across the subdomains; the communication strategy should be recomputed; and, possibly, the work rebalanced. This is a hard implementation task for distributed memory computers. In the current work, the Chimera method can be viewed as a preprocess, as the components will not change in time. Therefore, the parallelization is straightforward.

Just two specific aspects of the parallelization of the method deserve some attention. First, as it was mentioned in [17], the element graph used as an input for METIS should be based on the element-node connectivity and not element-face connectivity. In fact, the fringe nodes are connected to the extension nodes via elements and not faces. Second, if one wants to keep for some reason the hole elements and nodes in the mesh definition, one should be careful when assigning the weights to the element graph for METIS. In fact, if one puts a null weight on the hole elements, one subdomain 
could end up with all the hole elements. This is not a problem for computation purpose but can be a severe limitation in terms of memory.

\section{NUMERICAL EXAMPLES}

We now present some numerical examples to illustrate the performance but also some shortcomings of the Chimera method proposed in this work. In some of the following examples, manufactured solutions will be considered. They consist of manufactured velocity and pressure, $\boldsymbol{u}_{m}$ and $p_{m}$ respectively, with a given degree of smoothness. Then we seek for this solution by adding two force terms $f_{m}$ and $f_{c}$ to the momentum and continuity equations, respectively:

$$
\begin{gathered}
\rho \frac{\partial \boldsymbol{u}}{\partial t}+\rho(\boldsymbol{u} \cdot \nabla) \boldsymbol{u}-\nabla \cdot[2 \mu \boldsymbol{\varepsilon}(\boldsymbol{u})]+\nabla p=\boldsymbol{f}_{m}, \\
\nabla \cdot \boldsymbol{u}=f_{c},
\end{gathered}
$$

with

$$
\begin{gathered}
\boldsymbol{f}_{m}=\rho \frac{\partial \boldsymbol{u}_{m}}{\partial t}+\rho\left(\boldsymbol{u}_{m} \cdot \nabla\right) \boldsymbol{u}_{m}-\nabla \cdot\left[2 \mu \varepsilon\left(\boldsymbol{u}_{m}\right)\right]+\nabla p_{m}, \\
f_{c}=\nabla \cdot \boldsymbol{u}_{m},
\end{gathered}
$$

In addition, $\boldsymbol{u}_{m}$ is prescribed as a Dirichlet condition on the Dirichlet part of the boundary and the exact traction on the Neumann part of the boundary. If the flow is confined, the pressure is defined up to a constant. In this case, the pressure is prescribed to $p_{m}$ on one node of the domain. Note that if the manufactured solution belongs to the finite element space (linear in this case), the finite element solution is exactly the manufactured solution. We present here the selected examples and the specific characteristics of the method they want to illustrate:

- Matching overlap: Chimera = one-domain. To show that if the meshes in the overlap region match perfectly, then we recover the one-domain solution;

- Mesh convergence. To study the mesh convergence of the method.

- Mass conservation. Quantify the lack of mass conservation.

- Local refinement. To study the possibility to use the method as a mesh refinement technique. 
- Schur complement preconditioner. To compare the different Schur complement preconditioners presented in Section 5 .

- Wind farm. To test the performance method for a real application and show two possible ways of constructing the pyramid extensions.

\subsection{Matching overlap: Chimera $=$ one domain}

With this example we want to show that under certain circumstances, the solution of the Chimera method and the one-domain solution are exactly the same. The two conditions are:

- The mesh resulting from the union of the holed background and patch meshes is the same as the one-domain mesh.

- The extensions correspond exactly to the adjacent subdomain elements.

That is, the final meshes (each subdomain plus it respective extensions) match perfectly in the overlap region. Figure 18 shows the holed background (blue) and the patch (green) meshes with their respective extensions (red). Let us note that after the hole cutting in this example, the two subdomains are disjoint and their interfaces coincide.

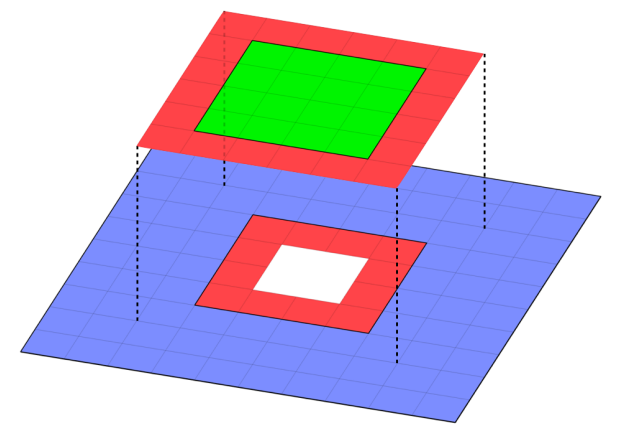

Figure 18. Matching overlap. Holed background (blue) and patch (green) meshes, and respective extensions (red). 
The one-domain and Chimera solutions should be the same regardless the flow problem. In the present case we choose the following simple manufactured solution on the unit square:

$$
\begin{aligned}
& \boldsymbol{u}_{m}=\left[x^{2}+y^{2}, x^{2}+y^{2}\right]^{t}, \\
& p_{m}=x^{2}+y^{2} .
\end{aligned}
$$

Figure 19 shows the velocity and pressure along a horizontal cut at mid height of the domain. The solutions coincide perfectly.
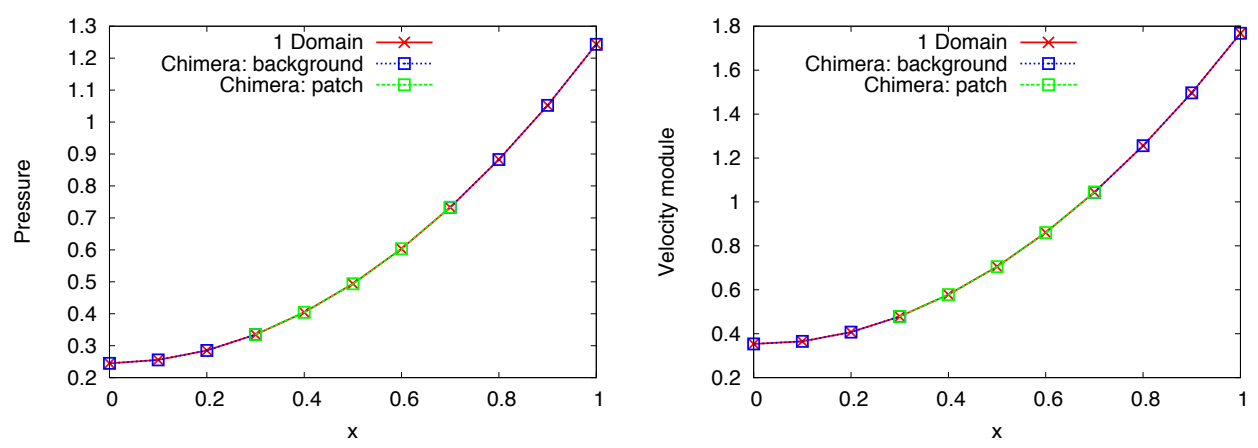

Figure 19. Matching overlap. Pressure and velocity module along horizontal cut.

\subsection{Mesh convergence}

The HERMESH method is exact if the solution belongs to the finite element space. That is, up to linear solution, the HERMESH method is nodally exact. Through this example we want to study the mesh convergence and to compare the results with the one-domain. To assess the mesh convergence we consider the following manufactured solution to be solved on the unit square:

$$
\begin{aligned}
& \boldsymbol{u}_{m}=\left[1+x+y^{2},-1-y-x^{2}\right]^{t}, \\
& p_{m}=x^{2}+y^{2} .
\end{aligned}
$$

Both the one-domain and Chimera method with two subdomains are solved, and two different scenarios are studied. In the present case, the two subdomains used for the Chimera method have the same mesh size and their interfaces are disjoint. The overlap comes from the extensions elements, after connecting the subdomains. On the one hand, the problem is solved with a Dirichlet condition 
on the velocity on all the boundaries. On the other hand, the right-hand side wall Dirichlet condition is substituted by a Neumann condition. Figure 20 shows the meshes and boundary conditions.

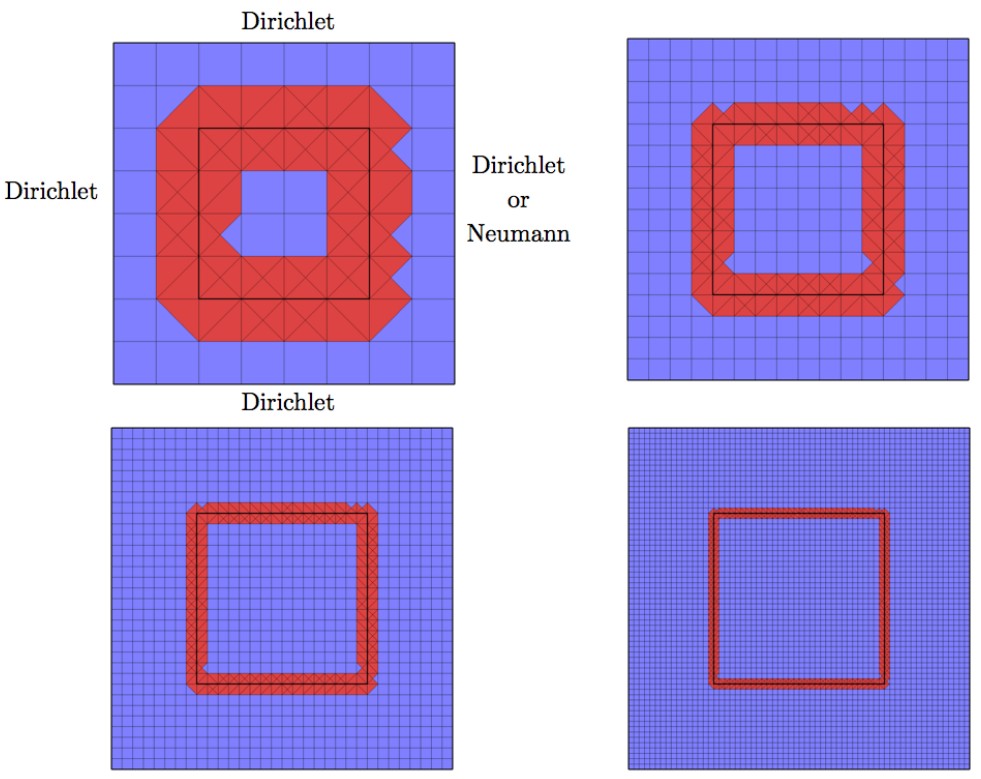

Figure 20. Mesh convergence. Meshes and boundary conditions.

As shown in earlier sections, the HERMESH coupling is equivalent to extending the subdomain and imposing a Dirichlet condition on the extension nodes. Therefore, we may not conserve mass at the subdomain interfaces. On the one hand, if the computational domain is confined, it is well known that the continuous problem does not have a solution (as the Dirichlet condition violates the data compatibility which establishes the global mass conservation). On the other hand, the numerical problem has a solution, but the pressure solution carries an error according to the magnitude of the mass imbalance. The problem can be avoided by imposing a Neumann condition on one of the walls. Figure 21 shows the errors obtained in velocity and pressure using one-domain and Chimera method, and for the Dirichlet and Neumann conditions. The expected rates of convergence are obtained, with low differences in velocity between the Chimera and one-domain solutions. The difference is much greater in pressure in the case of the Dirichlet condition on all the boundaries. 

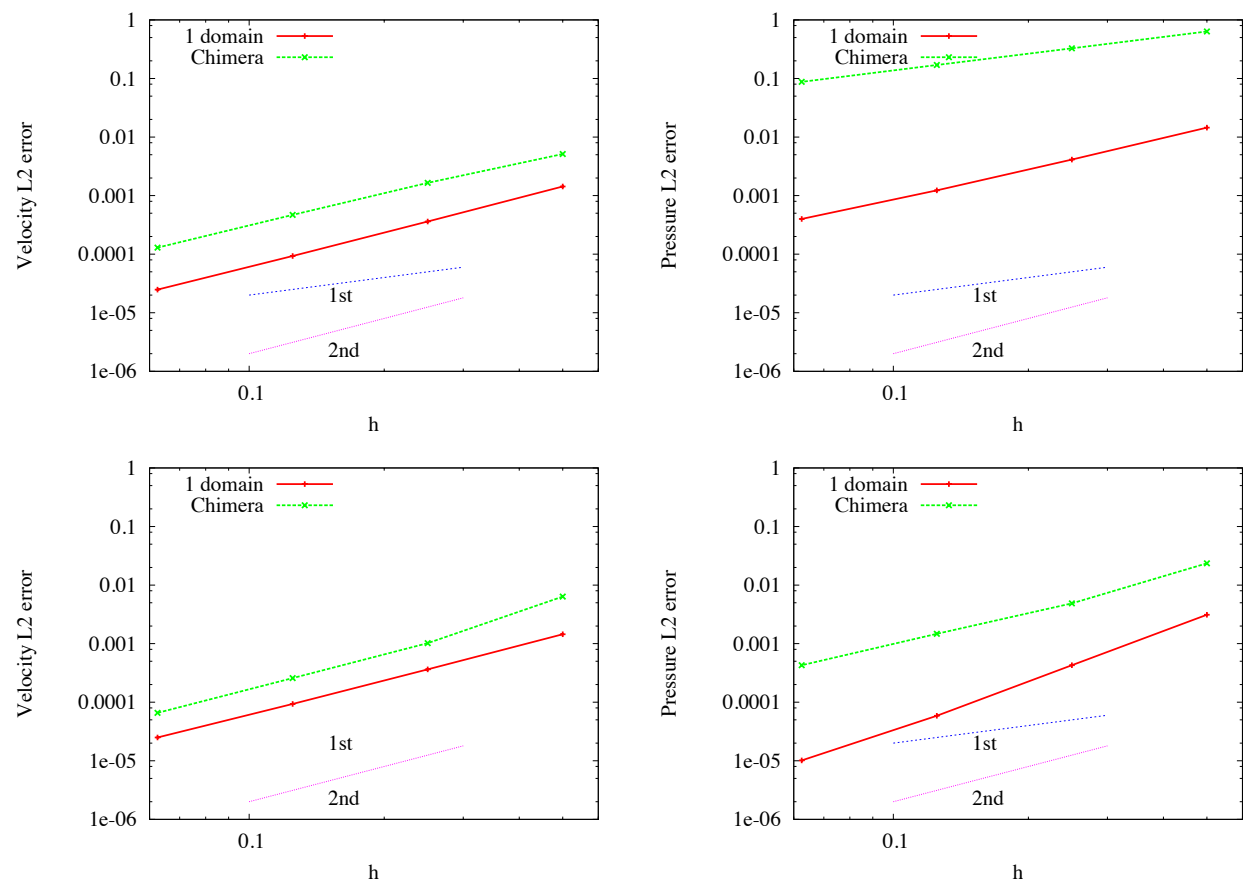

Figure 21. Mesh convergence. (Top) All Dirichlet conditions. (Bot.) Right wall is a Neumann condition. (Left) Velocity L2 residual. (Right) Pressure L2 residual.

\subsection{Local refinement}

In this example we present a possible application of the Chimera method: local refinement. To illustrate this application, the cavity flow at a Reynolds number 5000 is considered, on a unit square $[0,1] \times[0,1]$, solved on uniform and rather coarse meshes (finest one has only $50 \times 50$ elements) The Chimera method is compared to some one-domain solutions and with Ghia's results [41]. None of the meshes considered here is adapted to this high Reynolds number flow. What we want to show is that even if the core of the flow is not captured, the local refinement can help. For the Chimera method, the patch mesh is located on the bottom right corner, where a the flow exhibits a strong recirculation. Four solutions are going to be compared:

- Mesh 1: one-domain solution on a coarse mesh $(10 \times 10$ elements $)$.

- Mesh 2: one-domain solution on a fine mesh $(50 \times 50$ elements $)$. 
- Mesh 3: Chimera solution where the background and patch meshes are of the same size as Mesh 1 and Mesh 2, respectively.

- Mesh 4: Chimera solution where the background mesh is of the same size as Mesh1 and the patch is twice finer as Mesh2.

The four meshes are shown in Figure 22.

Mesh 1

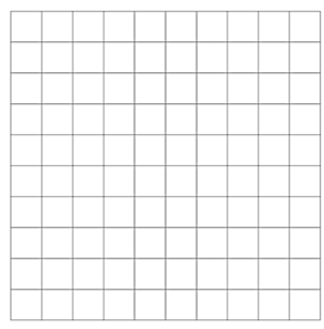

Mesh 3

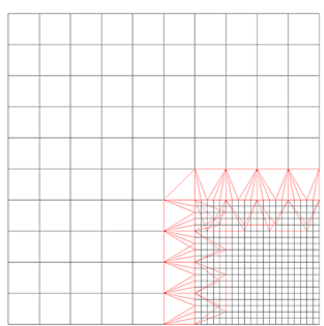

Mesh 2

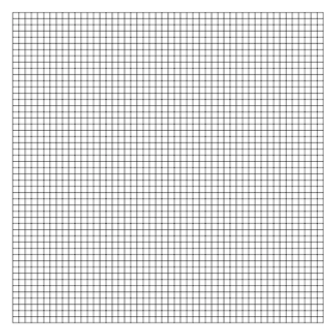

Mesh 4

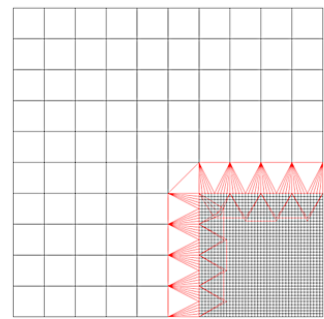

Figure 22. Local refinement. (Top) One-domain meshes (Mesh 1 and Mesh 2). (Bot.) Chimera meshes with extensions in red (Mesh 3 and Mesh 4).

Figure 23 shows the positive effects of the patch mesh to capture the recirculation. The solution was obtained with Mesh 3. Without using a patch mesh as a local refinement, no recirculation at all could be obtained, as only one very few nodes were located in the recirculation area.

Figure 24 compares the solutions obtained on one horizontal cut and one vertical cut. The coarse mesh (Mesh 1) does not capture the recirculation, partly captured by the fine mesh (Mesh 2). Both Chimera method enables to rectify the solution not only in the recirculation zone but also in the core of the cavity. We observe also that the very fine patch of Mesh 4 gives almost the same solution as Mesh 3: the error is dominated by the coarse mesh. 


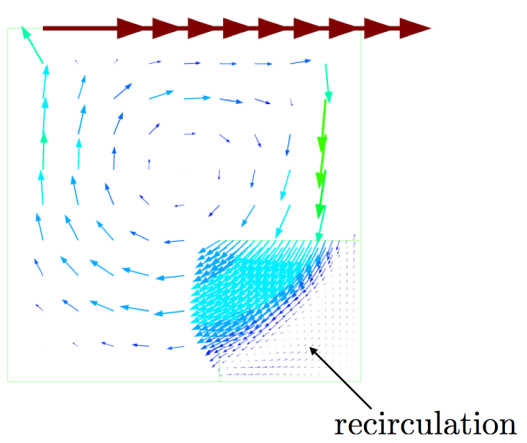

Figure 23. Local refinement. Recirculation captured by the patch mesh on Mesh 3.
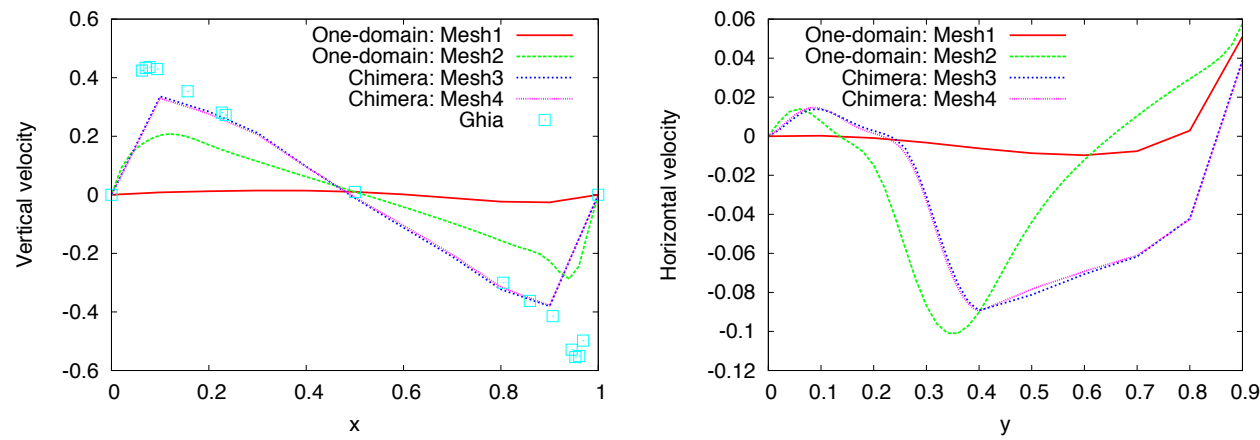

Figure 24. Local refinement. Comparison of solutions obtained on Mesh 1, Mesh 2, Mesh 3 and Mesh 4. (Left) Vertical velocity along horizontal cut at $y=0.5$. (Right) Horizontal velocity along horizontal cut at

$$
x=0.9 .
$$

\subsection{Schur complement preconditioner}

We showed in Section 5.2 that the coupling of the background and the patch through the extension elements leads to a nonsymmetric graph and therefore a nonsymmetric matrix for the pressure Schur complement preconditioner. In order to use symmetric iterative solvers like the CG or the DCG, the system should be symmetrized. Through this example we want to compare the four different strategies proposed in Section 5.2, namely all, symmetrized, zero, diag. The example is the flow over a square cylinder at Reynolds 10 based on the square length and inflow velocity. A symmetry condition is applied on top and bottom. 
We first compare the four convergence histories of the continuity equation. In this example, the Orthomin(1), linearization and time iterations are coupled. That is for each time step, only one Orthomin(1) and linearization iteration step is carried out. At iteration $k$ the residual is measured as

$$
\text { Continuity equation residual }=\frac{\left\|\mathbf{b}_{p}-\mathbf{A}_{p u}^{k} u^{k}-\mathbf{A}_{p p}^{k} p^{k}\right\|}{\left\|\mathbf{b}_{p}-\mathbf{A}_{p u}^{k} u^{k}\right\|}
$$

Figure 25 (Left) shows the mesh and extensions elements, and Figure 25 (Right) shows the convergence of the different options. We observe that assembling the whole extension is by far the best option. The same behavior has been observed in many other examples, so we conclude this is the best way to construct a symmetric preconditioner for the pressure Schur complement.
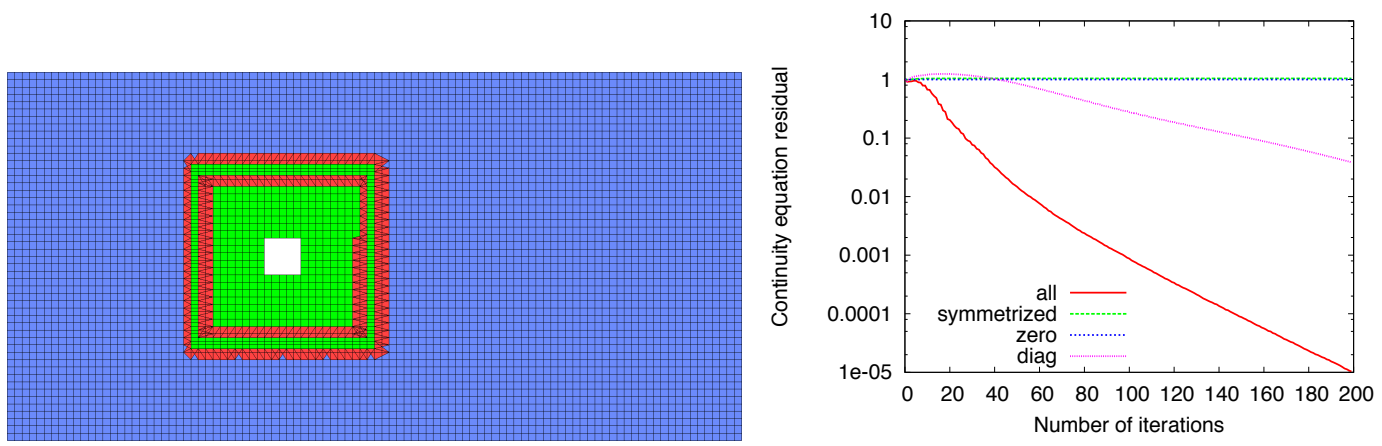

Figure 25. Schur complement preconditioner. (Left) Mesh. (Right) Convergence history of the continuity equation.

Let us observe the damping of the continuity equation residual. Figure 26 shows the evolution of this residual for some consecutive iterations, for both the diag-preconditioner (left column) and all-preconditioner (right column). We observe that with the first method, the error is much more concentrated near the interfaces and damped out much slower than with the all-preconditioner.

With respect to the fill-in of the matrix, Figure 27 illustrates the new connection obtained with the extension elements, for this example. 

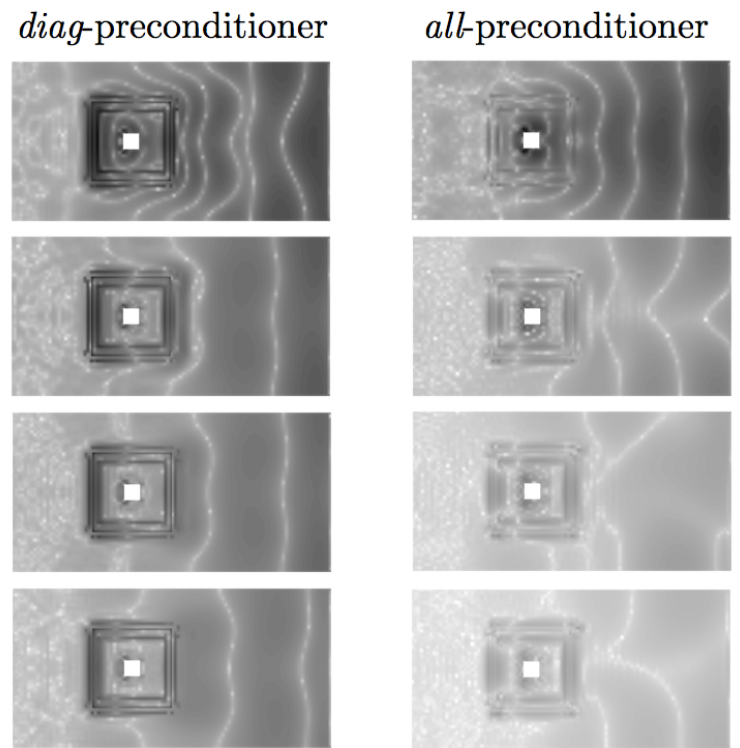

Figure 26. Schur complement preconditioner. Evolution of the residual of the continuity equation: diagpreconditioner in the left column and all-preconditioner in the right column.

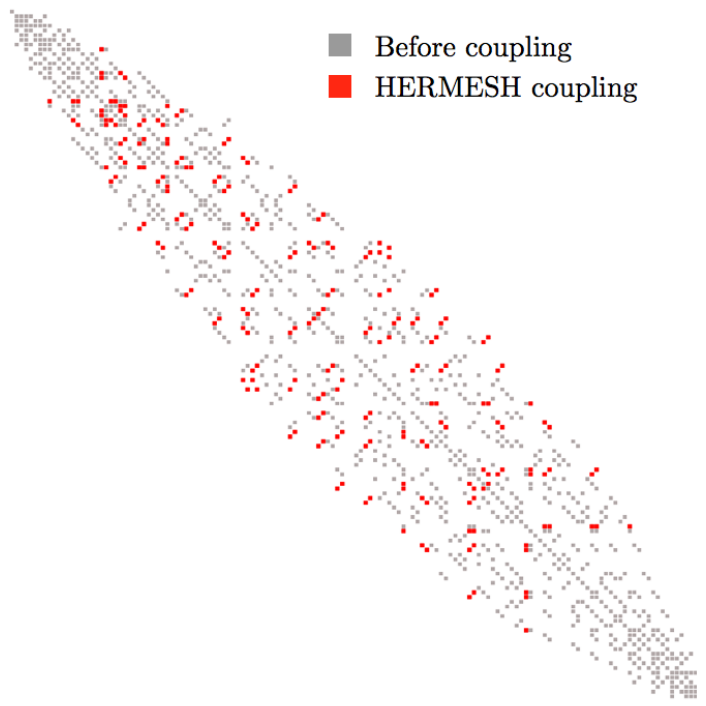

Figure 27. Schur complement preconditioner. All element contributions of extension elements are assembled. 


\subsection{Mass conservation}

A good review of this issue can be found in reference [42]. In the implicit strategy mentioned before [23], this question is also treated in details. In order to quantify the mass conservation, the NavierStokes equations are solved on the same geometry as in the mesh convergence example of Section 6.2, imposing a parabolic profile on the left wall (inflow), zero velocity on the top and bottom walls, and a zero traction condition on the right wall (outflow). Due to the not strictly conservative property of the HERMESH coupling, we expect that the background interpolates a global non-zero mass from the patch. The mesh convergence of the velocity is quadratic so it is expected that the mass imbalance is quadratic as well. This comment is confirmed by Figure 28, were we depict the difference in mass between inflow and outflow as well as the percentage of error.
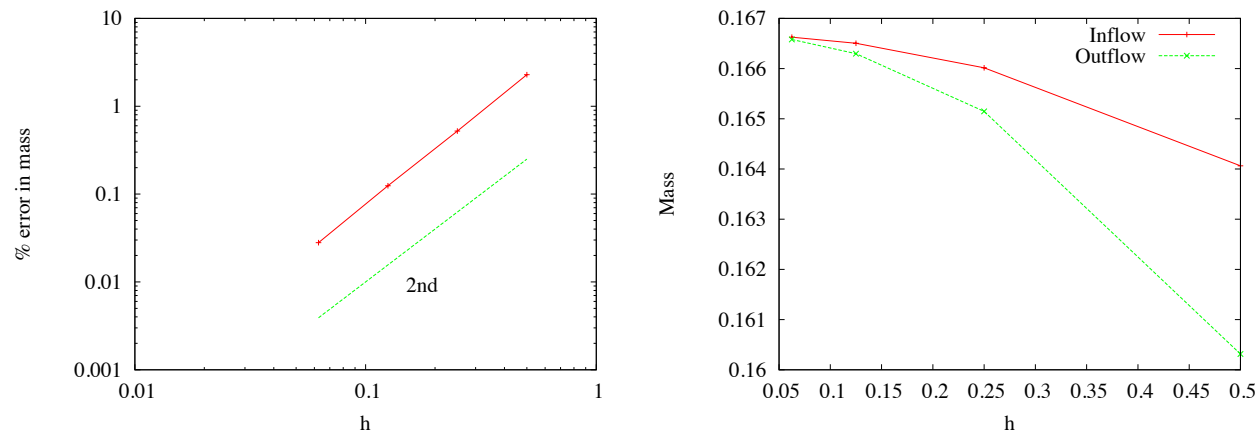

Figure 28. Mass conservation.

\subsection{Wind farm}

With this example we want to illustrate the extension from a hexahedra mesh to a tetrahedra mesh, applied to a wind farm simulation. In the current work the wind farm simulation consists in solving the Reynolds Averaged Navier-Stokes equations with a specific $k-\varepsilon$ turbulence model, on a real topography, using real wind measures as boundary conditions, and with an actuator disk model to account for the wind turbines. Other approximations like the virtual blade model are possible, see for example [43]. The actuator disk model consists simply in extracting volumetric momentum from the Navier-Stokes equations, according to some law involving the fluid velocity upstream of the disk. 
A good review of computational fluid dynamics for wind turbine wake aerodynamics simulations can be found in [44]. Up to the author's knowledge, the application of Chimera methods to simulate micro-scale wind farms is new, although the Chimera method has been used to simulate single wind turbines [45].

The cost-effectiveness of a wind farm is mainly driven by the cost of the terrain, management of the infrastructure, and the power generated by the wind turbines. In order to reduce the infrastructural costs, one would therefore like to increase the density of wind turbines on the terrain. The problem is that each of the turbines causes a shielding effect in its neighboring due to the presence of the downstream wake. For this reason, the turbines inside the wind farm have to be located in such way that these effects are minimized, and thus the associated power maximized. The application of the Chimera method in this problem consists in having an independent patch for each turbine and coupling all of them to the background mesh which contains the topography. Due to the $\log$ profile of the flow velocity and rapidly decreasing $\varepsilon$ away from the wall, a boundary layer mesh is unavoidable. Figure 29 illustrates the idea, where 28 patches were superimposed on a structured background mesh attached to the topography.
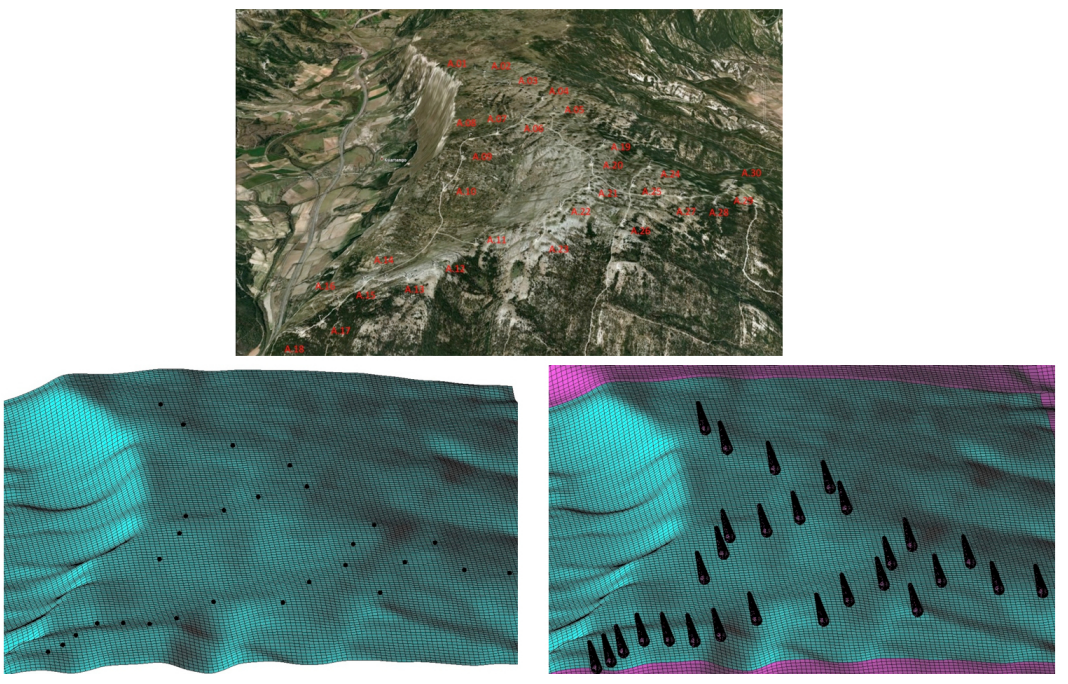

Figure 29. Wind Farm. Example of topography and patches with actuator disks. 
Typically, the background mesh for these problems consists of hexahedra with a boundary layer refinement near the ground. This implies another issue in the creation of the extension elements. First, the extension elements that connect the background mesh with the patch meshes are pyramids because the outer boundary of the hole is formed by quadrilaterals. Second, as some quadrilaterals of the hole boundary may come from an anisotropic hexahedra, one could require the pyramids to inherit the hexahedra aspect ratio. Many references about the anisotropy question can be found in the literature $[46,47,48]$. Figure 30 illustrates the two ways of creating the extension elements from the structured background mesh to the nearly isotropic patch mesh. On the one hand, the left part of the figure shows the isotropic pyramid extension elements. On the other hand, the right part of the figure shows pyramids which shape respect the background boundary layer mesh.

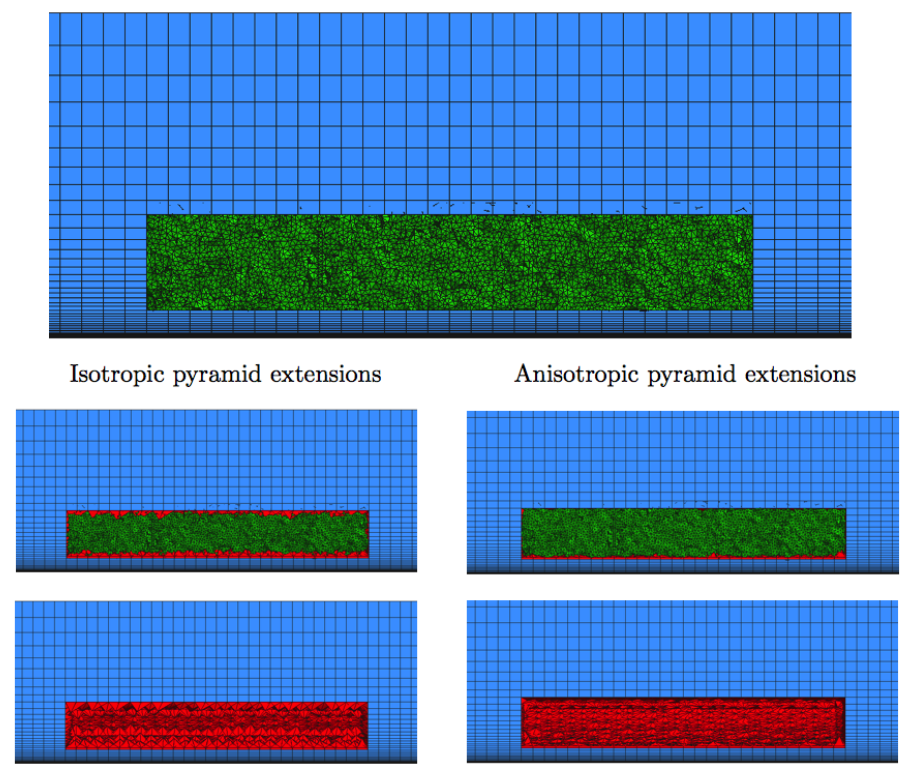

Figure 30. Wind farm. Extension from background to patch. (Top) Meshes. (Left) Isotropic pyramids. (Right) Anisotropic pyramids to mimic the boundary layer.

Figure 31 illustrates the isotropic case. The right part of the figure represents the hole together with the extension elements of a corner fringe node. The left part of the figure shows a zoom on these isotropic extension elements. 


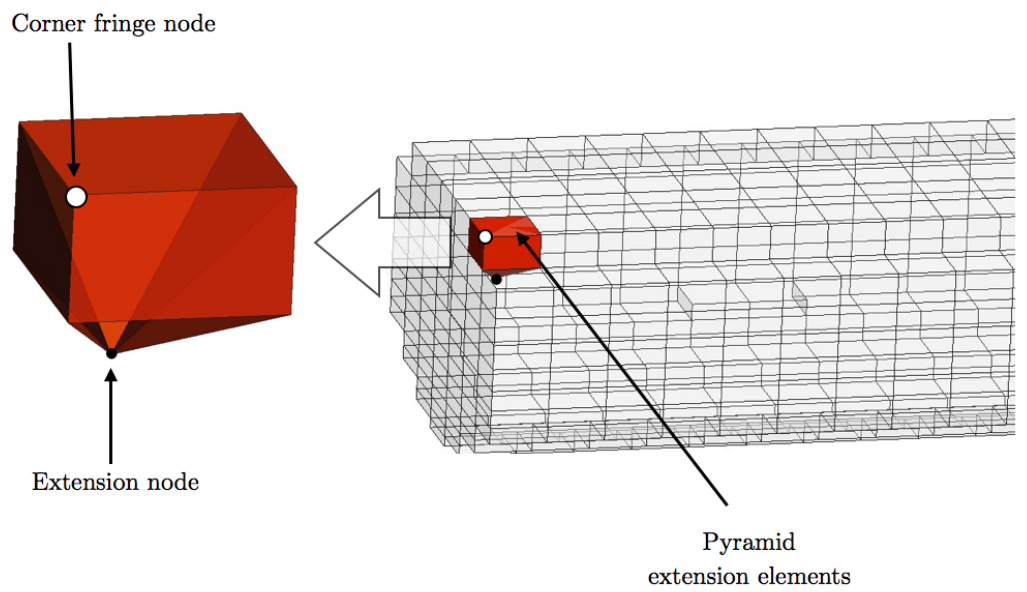

Figure 31. Wind farm. (Left) Pyramid extension elements from on corner fringe node of the background. (Right) background hole and pyramid extension element.

Figure 32 shows the results obtained for two wind turbines, using isotropic pyramids, on a flat topography. The second turbine is located 2.5 rotor diameters downstream of the first one. The top part of the figure shows the patch boundary meshes. The other figures shows the solution (velocity, pressure and turbulent viscosity) obtained on a vertical cut passing through the middle of the actuator disk.

Finally, we have compared the one-domain solution, obtained on a structured mesh, with the Chimera method for a single wake case, extracted from the experimental Sexbierum test [49]. In Figure 33 we can see how both cases, one-domain and Chimera domain reproduce very similar results. It is worth mentioning that the number of nodes of the one-domain mesh is 290,835 while 156,740 were sufficient in the case of the Chimera method. The difference comes from the fact that lots of elements are useless when capturing the wind turbine on the structured mesh of the one-domain solution. This difference in number of nodes can be significant when considering tens of wind turbines. Figure 33 represent the velocity, pressure and turbulent viscosity for the case of 

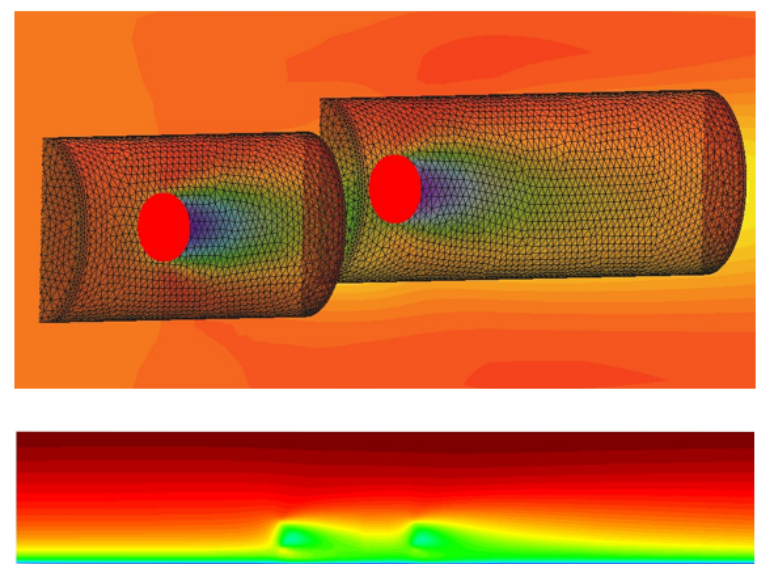

\section{Velocity}

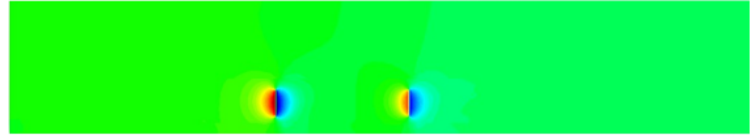

Pressure

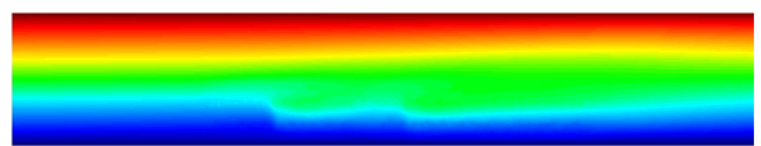

Turbulent viscosity

Figure 32. Wind farm. Patch meshes and solution.

2 turbines located in one after the other and separated 2.5 diameters of the rotor. The solution are almost coinciding.

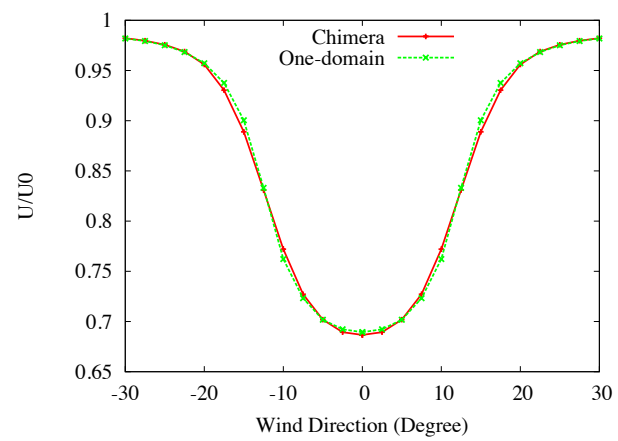

Figure 33. Wind farm. Wind speed deficit 2.5D downstream. Comparison between one-domain and Chimera method. 


\section{CONCLUSIONS}

The Chimera method presented in [20] was extended to the solution of the Navier-Stokes equations and its performance was enhanced. With respect to the original reference, the following new aspects have been implemented or treated:

- A new and robust hole cutting to avoid non-manifold interfaces;

- Extension from a hexahedra mesh using pyramids;

- Implementation aspects for Navier-Stokes;

- Symmetrization of the pressure Schur complement preconditioner;

- Chimera for local refinement.

Through some examples, the authors demonstrated the reliability and shortcomings of the method. Although the method is not fully conservative, the error in mass conservation is consistent with the velocity mesh convergence. The presented implementation is now used in daily production by the authors as a preprocess method to treat fixed components. The next development will consist in implementing the coupling after the mesh partitioning, in a distributed memory environment, in order to deal with moving components.

\section{ACKNOWLEDGEMENTS}

This work has been partly carried out in the framework of the FP7 European projects W2Plastics. Part of the work of Beatriz Eguzkitza was sponsored by an IBERDROLA grant

\section{REFERENCES}

1. Steger J, Benek FDJ. A chimera grid scheme. Advances in Grid GEneration 1983; 5:59-69.

2. Benek J. Chimera. a grid-embedding technique. Technical Report, DTIC Document 1986.

3. Steger J, Benek J. On the use of composite grid schemes in computational aerodynamics. Comp. Meth. Appl. Mech. Eng. 1987; 64:301-320.

4. Chattot J, Wang Y. Improvement treatment of intersecting bodies with the chimera method and validation with a simple fast flow solver. Computer \& Fluids 1998; 27:721-740. 
5. Zheng Y, Liou MS. A novel approach of three-dimensional hybrid grid methodology: Part 1. grid generation. Comp. Meth. Appl. Mech. Eng. 2003; 192:4147-4171.

6. Kao KH, Liou MS, Chow CY. Grid adaptation using chimera composite overlapping meshes. AIAA journal 1994; 32(5):942-949.

7. Meakin, Robert L. An efficient means of adaptive refinement within systems of overset grids. AIAA paper 1995; 1722:1995.

8. Meakin, Robert L. Moving body overset grid methods for complete aircraft tiltrotor simulations. AIAA paper 1993; 3350:1993.

9. Prewitt NC, Belk DM, Shyy W. Parallel computing of overset grids for aerodynamic problems with moving objects. Progress in Aerospace Sciences 2000; 36(2):117-172.

10. Blades EL, Marcum D. A sliding interface method for unsteady unstructured flow simulations. Int. J. Num. Meth. Fluids 2007; 53(3):507-529.

11. Wolf C. A chimera simulation method and detached eddy simulation for vortex-airfoil interactions. PhD Thesis, Georg-August-Universität Göttingen 2011.

12. Nielsen E, Diskin B. Discrete adjoint-based design for unsteady turbulent flows on dynamic overset unstructured grids. AIAA Journal 2013; 51(6): 1355-1373.

13. Noack R. Suggar: a general capability for moving body overset grid assembly. AIAA paper 2005; 5117:2005.

14. Rogers S, Suhs N, Dietz W. Pegasus 5: an automated preprocessor for overset-grid computational fluid dynamics. AIAA journal 2003; 41(6):1037-1045.

15. DLBrown, Henshaw W, Quinlan D. Overture: Object-oriented tools for overset grid applications. AIAA paper No. 99 1999; 9130.

16. Fournier Y, Benhamadouche S, Monfort D, Laurence D. Non conforming meshes and rans/les coupling: two challenging aims for a CFD code. HT-FED-2004, 2004 ASME Heat Transfer/Fluids Engineering Summer Conference, Charlotte (USA), 2004.

17. Eguzkitza B, Houzeaux G, Aubry R, Vázquez M. A parallel coupling strategy for the chimera and domain decomposition methods in computational mechanics. Computers \& Fluids 2013; 80:128-141.

18. Park S, Jeong B, Lee J, Shin H. Hybrid grid generation for viscous flow analysis. Int. J. Num. Meth. Fluids 2012; .

19. Behr M, Tezduyar T. The shear-slip mesh update method. Comp. Meth. Appl. Mech. Eng. 1999; 174:261-274.

20. Houzeaux G, Codina R. A Chimera method based on a Dirichlet/Neumann(Robin) coupling for the Navier-Stokes equations. Comp. Meth. Appl. Mech. Eng. 2003; 192(31-32):3343 - 3377.

21. Quarteroni A, Valli A. Domain decomposition methods for partial differential equations. Oxford University Press, USA, 1999.

22. Rome C, Glockner S. An implicit multiblock coupling for the incompressible navier-stokes equations. Int. J. Num. Meth. Fluids 2005; 47(10-11):1261-1267.

23. Ahusborde E, Glockner S. An implicit method for the navier-stokes equations on overlapping block-structured grids. Int. J. Num. Meth. Fluids 2010; 62(7):784-801. 
24. Burton TM, Eaton J. Analysis of a fractional-step method on overset grids. J. Comput. Phys. 2002; 177(2):336-364.

25. Houzeaux G, Vázquez M, Aubry R, Cela J. A massively parallel fractional step solver for incompressible flows. $J$. Comput. Phys. 2009; 228(17):6316-6332.

26. Dey S, Shephard M, Georges M.Elimination of the adverse effects of small model features by the local modification of automatically generated meshes. Eng. with Comp. 1997; 13(3):134-152.

27. Khamayseh A, Kuprat A. Deterministic point inclusion methods for computational applications with complex geometry. Computational Science \& Discovery 2008; 1.

28. Batchelor G. An Introduction to Fluid Dynamics. Cambridge University Press, 1970.

29. Houzeaux G, Principe J. A variational subgrid scale model for transient incompressible flows. Int. J. Comp. Fluid Dyn. 2008; 22(3):135-152.

30. Houzeaux G, Aubry R, Vázquez M. Extension of fractional step techniques for incompressible flows: The preconditioned orthomin(1) for the pressure schur complement. Computers \& Fluids 2011; 44:297-313.

31. Golub G, Loan CV. Matrix Computations. The Johns Hopkins University Press, 1996.

32. Greenbaum A. Iterative Methods for Solving Linear Systems. SIAM series in frontiers in Applied Mathematics, 1997.

33. Metis, family of multilevel partitioning algorithms. URL http://glaros.dtc . umn . edu/gkhome/views /metis.

34. Saad Y. Iterative Methods for Sparse Linear Systems. SIAM, 2003.

35. Aubry R, Mut F, Löhner R, Cebral J. Deflated preconditioned conjugate gradient solvers for the pressure-poisson equation. J. Comput. Phys. 2008; 227(24):10 196-10 208.

36. Soto O, Löhner R, Camelli F. A linelet preconditioner for incompressible flow solvers. Int. J. Num. Meth. Heat Fluid Flow 2003; 13(1):133-147.

37. Lohner R, Mut F, Cebral J, Aubry R, Houzeaux G. Deflated preconditioned conjugate gradient solvers for the pressure-poisson equation: Extensions and improvements. Int. J. Num. Meth. Eng. 2011; 87:2-14.

38. Djomehri M, Biswas R. Performance enhancement strategies for multi-block overset grid cfd applications. Parallel Computing 2003; 29(11):1791-1810.

39. Meakin R, Wissink A. Unsteady aerodynamic simulation of static and moving bodies using scalable computers. Proc. 14th AIAA Computational Fluid Dynamics Conference, 1999.

40. Sitaraman J, Floros M, Wissink A, Potsdam M. Parallel domain connectivity algorithm for unsteady flow computations using overlapping and adaptive grids. J. Comput. Phys. 2010; 229(12):4703-4723.

41. Ghia U, Ghia K, Shin C. High-Re solutions for incompressible flow using the Navier-Stokes equations and a multigrid method. J. Comput. Phys. 1982; 48:387-411.

42. Liu J, Shyy W. Assessment of grid interface treatments for multi-block incompressible viscous flow computation. Computers \& Fluids 1996; 25(8):719-740.

43. Hussein A, El-Shishiny H. Modeling and simulation of micro-scale wind farms using high performance computing. Int. J. Comp. Meth. 2012; 9(02). 
44. Sanderse B, der Pijl S, BKoren. Review of computational fluid dynamics for wind turbine wake aerodynamics. Wind Energy 2011; 14(7):799-819.

45. Zahle F, Sørensen N, Johansen J. Wind turbine rotor-tower interaction using an incompressible overset grid method. Wind Energy 2009; 12(6):594-619.

46. Knupp P. Algebraic mesh quality metrics. SIAM J. Sci. Comput. 2001; 23(1):193-218.

47. Frey P, Alauzet F. Anisotropic mesh adaptation for cfd computations. Comp. Meth. Appl. Mech. Eng. 2005; 194(48):5068-5082.

48. George P, Borouchaki H. Génération automatique de maillages tridimensionnels respectant une carte de taille. Revue Europénne des Éléments 1998; 7(4):339-363.

49. Cleijne J. Results of sexbierum wind far. Report 92-388 1992; 1. 